\title{
Altered pattern of circulating miRNAs in HIV lipodystrophy perturbs key adipose differentiation and inflammation pathways
}

\author{
Suman Srinivasa, ${ }^{1}$ Ruben Garcia-Martin, ${ }^{2}$ Martin Torriani, ${ }^{3}$ Kathleen V. Fitch, ${ }^{1}$ Anna R. Carlson, ${ }^{1}$ \\ C. Ronald Kahn, ${ }^{2}$ and Steven K. Grinspoon ${ }^{1}$ \\ ${ }^{1}$ Metabolism Unit, Massachusetts General Hospital and Harvard Medical School, Boston, Massachusetts, USA. ${ }^{2}$ Section \\ on Integrative Physiology and Metabolism, Joslin Diabetes Center and Harvard Medical School, Boston, Massachusetts \\ USA. ${ }^{3}$ Division of Musculoskeletal Imaging and Intervention, Massachusetts Ceneral Hospital and Harvard Medical School, \\ Boston, Massachusetts, USA.
}

We identified a microRNA (miRNA) profile characterizing HIV lipodystrophy and explored the downstream mechanistic implications with respect to adipocyte biology and the associated clinical phenotype. miRNA profiles were extracted from small extracellular vesicles (sEVs) of HIV-infected individuals with and without lipodystrophic changes and individuals without HIV, among whom we previously showed significant reductions in adipose Dicer expression related to HIV. miR-20a$3 p$ was increased and miR-324-5p and miR-186 were reduced in sEVs from HIV lipodystrophic individuals. Changes in these miRNAs correlated with adipose Dicer expression and clinical markers of lipodystrophy, including fat redistribution, insulin resistance, and hypertriglyceridemia. Human preadipocytes transfected with mimic miR-20a-3p, anti-miR-324-5p, or anti-miR-186 induced consistent changes in latent transforming growth factor beta binding protein 2 (Ltbp2), Wisp2, and $\mathrm{Neb}$ expression. Knockdown of Ltbp2 downregulated markers of adipocyte differentiation (Fabp4, Ppary, C/ebpa, Fasn, adiponectin, Glut4, CD36), and Lamin C, and increased expression of genes involved in inflammation (IL1/, IL6, and CcI20). Our studies suggest a likely unique sEV miRNA signature related to dysregulation of Dicer in adipose tissue in HIV. Enhanced miR-20a-3p or depletion of miR-186 and miR-324-5p may downregulate Ltbp2 in HIV, leading to dysregulation in adipose differentiation and inflammation, which could contribute to acquired HIV lipodystrophy and associated metabolic and inflammatory perturbations.

Authorship note: SS and RGM contributed equally to this work.

Conflict of interest: CRK received consulting fees from Kaleido Biosciences, Cellarity, ERX Therapeutics, and CohBar. SKC has received research funding from KOWA, Gilead, Viiv, and Theratechnologies and received consulting fees from Theratechnologies and Viiv.

Copyright: (c) 2021, Srinivasa et al. This is an open access article published under the terms of the Creative Commons Attribution 4.0 International License.

Submitted: April 9, 2021

Accepted: August 11, 2021

Published: September 22, 2021

Reference information: JCI Insight. 2021;6(18):e150399.

https://doi.org/10.1172/jci.

insight.150399.

\section{Introduction}

HIV lipodystrophy is the most prevalent form of acquired lipodystrophy (1). The altered fat distribution seen in HIV lipodystrophy has significant implications for metabolic risk, including cardiovascular disease $(2,3)$ and nonalcoholic fatty liver disease $(4)$, which are leading contributors to morbidity and mortality in HIV. Individuals with HIV lipodystrophy can present with lipohypertrophy, with increased fat in the abdomen, viscera, and dorsocervical areas, and/or lipoatrophy, with loss of fat in the face, extremities, and subcutaneous depots. These changes are usually associated with insulin resistance, metabolic dysregulation, and inflammation. The pathogenic mechanisms of HIV lipodystrophy remain unclear. While HIV lipodystrophy was initially attributed to antiretroviral-mediated toxic effects on adipocytes, there is an increasing appreciation that the HIV and its related proteins may have direct effects on key metabolic and inflammatory pathways that may impact adipose function (5). Recent progress by our group has focused on a mechanistic hypothesis for adipose dysfunction and metabolic changes in HIV, relating to the dysregulation of Dicer (6), an integral protein in the microRNA (miRNA) processing pathway, and its subsequent effects on critical miRNAs regulating adipogenic pathways $(7,8)$.

miRNAs have important regulatory functions in adipose tissue biology. Indeed, mice lacking Dicer in adipocytes (Adiponectin-Cre+Dicer ${ }^{\mathrm{f} / \mathrm{fl}}$; aDicer-KO) display a marked biological dysregulation of multiple miRNAs and mRNA targets associated with lipodystrophy and demonstrate a phenotype composed of multiple metabolic alterations, including insulin resistance, mitochondrial dysfunction, signs of oxidative stress, fatty liver, and premature mortality $(8,9)$. These phenotypic changes in the aDicer-KO mice closely resemble those seen 
in the lipodystrophy associated with HIV (8). Interestingly, expression of Dicer in subcutaneous adipose tissue is markedly reduced in individuals with HIV lipodystrophy, providing a mechanistic link for overlapping metabolic features between HIV lipodystrophy and the aDicer-KO mice $(6,8)$.

Given the downregulation of adipose Dicer in individuals with HIV lipodystrophy, a human physiological model that complements the animal data from the complete genetic knockout, we sought to explore whether these individuals with lipodystrophy demonstrated alterations in circulating miRNAs that might underlie the metabolic alterations in lipodystrophy $(3,10-12)$. Adipose tissue is a major contributor to the circulating miRNAs carried by small extracellular vesicles (sEVs), which have regulatory functions in distant tissues $(13,14)$. Building on prior work $(13)$, we now comprehensively assess circulating miRNAs in individuals with HIV and lipodystrophy, individuals with HIV without lipodystrophy, and matched uninfected individuals and perform functional studies to investigate the subsequent effects of altered miRNAs on adipose tissue. We characterize a unique pattern of differentially regulated sEV miRNAs, which we show to have important gene targets in adipocytes, affecting critical adipogenic and inflammatory pathways. Thus, the current investigation identifies a potentially novel mechanism for acquired HIV lipodystrophy, highlighting key downstream consequences of reduced Dicer expression, systemic dysregulation of key miRNAs, and subsequent effects of altered miRNAs on genes affecting adipose regulation.

\section{Results}

Altered body composition and reduced adipose Dicer expression seen in HIV lipodystrophy. To address the role of the miRNA processing pathway in the metabolic alterations caused by HIV infection, we first studied a cohort of individuals with HIV displaying lipodystrophy, individuals with HIV and no lipodystrophy, and uninfected individuals without HIV. As shown in Table 1, age and race were similar upon stratification into 3 groups (HIV/lipo, HIV/nonlipo, non-HIV). Dorsocervical adipose tissue area was largest in the HIV/lipo group and smallest in the non-HIV group $(P<0.004)$. These dorsocervical adipose tissue measurements obtained by imaging confirmed our clinical assessment for lipodystrophy in which participants with lipodystrophy were recruited for presence of increased dorsocervical fullness (Table 1). In addition, we confirmed using dual-energy X-ray absorptiometry (DXA) that limb fat $(6345.4 \pm 975.2$ vs. $8679.7 \pm 816.9 \mathrm{~g}, P=0.04$ by 1-way ANOVA) was lower in the HIV lipodystrophic versus HIV nonlipodystrophic phenotypes, suggesting evidence of peripheral lipoatrophy as would be expected in lipodystrophy. As previously described (6), Dicer expression, as determined by quantitative PCR (qPCR), was most reduced among HIV/lipo, followed by HIV/nonlipo and non-HIV $(2.49[0.02,4.88]$ vs. $11.20[4.83,21.45]$ vs. $17.69[10.72,47.91]$, median and IQR, $P=0.002$ ) (Table 1). HIV-related characteristics among HIV/lipo participants and HIV/nonlipo participants differed by duration of HIV infection ( $24 \pm 2$ vs. $18 \pm 3$ years, $P=0.07)$, duration of ART ( $20 \pm 2$ vs. $11 \pm 2$ years, $P=0.007)$, duration of protease inhibitor use ( $16 \pm 3$ vs. $9 \pm 2$ years, $P=0.08)$, and CD $8^{+}$ count $(725 \pm 115$ vs. $1260 \pm 164$ cells $/ \mu \mathrm{L}, P=0.02)$ (all ranges show mean \pm SEM). Overall, both HIV groups demonstrated good immunological control based upon $\mathrm{CD}^{+}$count and had similar viral loads. Respiratory quotient determined by indirect calorimetry was lowest among the non-HIV participants (Table 1). Among the HIV participants, Dicer expression was significantly related to $\mathrm{CD}^{+} P=0.51, P=0.03$ ) count and inversely to duration of $\operatorname{ART}(P=-0.60, P=0.009)$.

Altered pattern of circulating $s E V$-carried miRNAs in HIV lipodystrophy associated with reduced adipose Dicer expression. As we have previously demonstrated, reduced adipose tissue Dicer in mice leads to changes in the expression levels of circulating sEV miRNAs (13). Given the reduction in adipose Dicer expression in HIV patients with lipodystrophy, we addressed whether circulating sEV miRNAs were also changed in these individuals. To do so, we isolated serum sEV using differential centrifugation protocol (15) from HIV/lipo, $\mathrm{HIV} /$ nonlipo, and non-HIV participants (Figure 1A). This protocol led to vesicles in the expected range for exosomes (50-200 nm), enriched in the sEV markers CD63 and TSG101, while depleted from the cellular marker calnexin (Supplemental Figure 1, A-C; supplemental material available online with this article; https://doi.org/10.1172/jci.insight.150399DS1). As shown in Figure 1B, each group (non-HIV, HIV/nonlipo, and HIV/lipo) displayed a distinct circulating sEV miRNA profile. Comparing the HIV/lipo and nonHIV participants, the levels of $7 \mathrm{sEV}$ miRNAs were significantly upregulated while 12 were downregulated (Figure 2, A and B). In the comparison of HIV/nonlipo with non-HIV, $30 \mathrm{sEV} \mathrm{miRNAs} \mathrm{were} \mathrm{significant-}$ ly upregulated and 27 were downregulated (Figure 2, A and B). The miRNA profile of HIV participants with lipodystrophy was also compared with HIV participants without lipodystrophy. In this comparison, the expression of 33 miRNAs was significantly upregulated and 35 downregulated (Figure 2, A and B). 
Table 1. Baseline demographic and clinical characteristics among HIV/lipodystrophy, HIV/nonlipodystrophy, and non-HIV participants

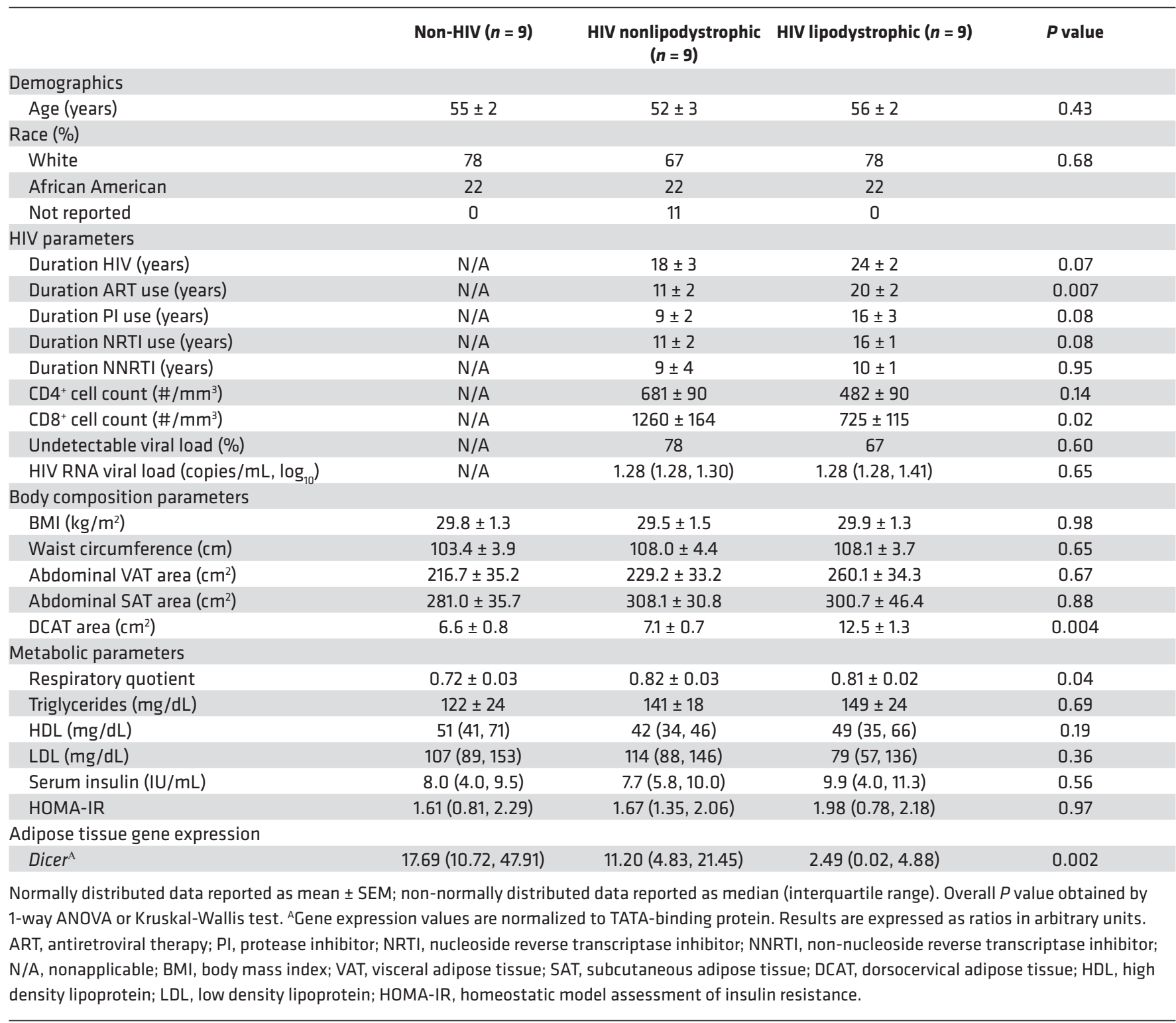

Interestingly, although additional changes did not reach statistical significance, expression of many different miRNAs was generally decreased in the HIV/lipo group as observed by the left shift in the base of the volcano plots shown in Figure 2A and the bluish tone of the upper half column for HIV/lipo in the heatmap displayed in Figure 2B. This observation is in line with a previous observation from our group (13) and highlights that many of the differentially expressed miRNAs were downregulated. Thus, HIV infection either with or without lipodystrophy leads to significant changes in multiple circulating sEV miRNAs. Decreases in the circulating sEV miRNA population were also seen that specifically associated with lipodystrophy.

The top differentially regulated miRNAs are shown in Figure 2B and can be clustered in 3 major groups: sEV miRNAs reduced in HIV lipodystrophy, others reduced in HIV without clinical evidence of lipodystrophy, and others elevated in HIV regardless of a lipodystrophy presentation. Assessing for differences using a more stringent statistical approach (FDR < 0.05) revealed miR-20a-3p, miR-324-5p, and miR-186 as the top changed miRNAs. Notably, miR-20a-3p showed a stepwise progression and was significantly elevated in HIV/nonlipo compared with non-HIV and even further elevated in HIV/lipo (Figure 2C). In contrast, miR-324-5p and miR-186 were specifically downregulated in the HIV/lipo group compared with the other groups (Figure 2C). Among all participants, upregulation of miR-20a-3p correlated with downregulation 
A

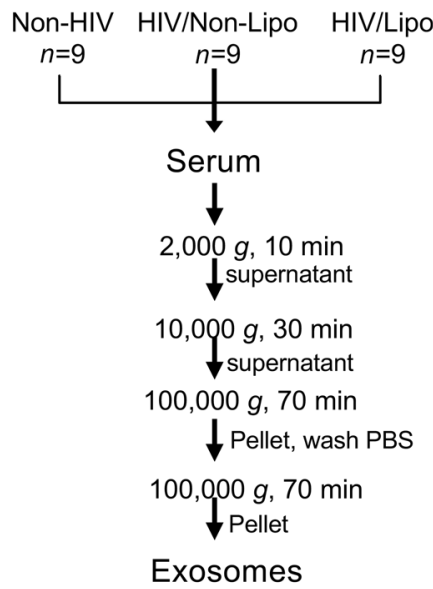

B

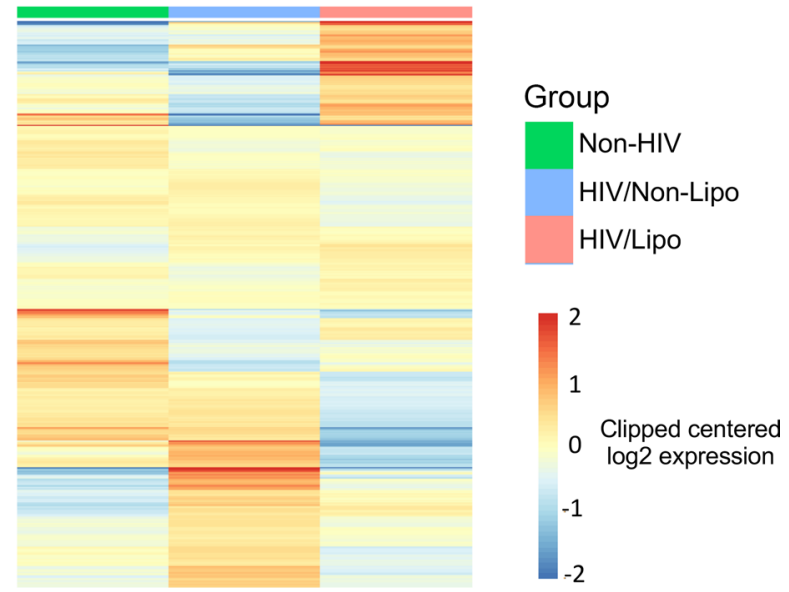

Figure 1. Isolation of circulating sEV miRNAs correlating to HIV infection and lipodystrophy. (A) Diagram representing the sEV isolation method. Exosomes were isolated from HIV/lipodystrophy, HIV/nonlipodystrophy, and non-HIV participants ( $n=9$ per group) by differential centrifugation protocol and subjected to qPCR-based miRNA profiling. (B) Heatmap showing the group average expression for each miRNA measured in serum. Red color indicates high expression; blue indicates low expression. Left column (non-HIV, indicated by green box at top); middle column (HIV nonlipo, indicated by blue box at top); right column (HIV lipo, indicated by pink box at top). $n=8-9$ per group applies to all the panels in this figure.

of miR-324-5p ( $\rho=-0.71, P=0.002)$ and miR-186 $(\rho=-0.74, P=0.002)$. In addition, downregulation of miR-324-5p correlated with downregulation of miR-186 ( $\rho=0.54, P=0.006)$ (Table 2). Interestingly, expression of these circulating miRNAs in sEVs correlated significantly to adipose Dicer expression among all participants (miR-20a-3p, $\rho=-0.61, P=0.01$; miR-324-5p, $\rho=0.39, P=0.05$; miR-186, $\rho=0.53, P=$ 0.008 ) (Table 3 ) and also strongly correlated with dorsocervical adipose tissue area (our clinical measure of lipodystrophy) (Table 3). Levels of miR-20a-3p correlated with respiratory quotient ( $\rho=-0.64, P=0.008$ ), triglycerides ( $\rho=0.52, P=0.04)$, insulin $(\rho=0.67, P=0.005)$, and HOMA-IR $(\rho=0.50, P=0.047)$, while miR-186 related to reduced $\mathrm{CD}^{+}$cell count $(\rho=0.55, P=0.04)$ (Table 3$)$. Taken together, these data suggest that the alteration in the levels of circulating sEV-carried miRNAs, i.e., miR-20a-3p, miR-186, and miR-324-5p, might serve as markers for the degree of lipodystrophy among individuals with HIV.

Altered pattern of circulating sEV-carried miRNAs in HIV lipodystrophy is concordant with changes in aDicer-KO mice and transfection with Dicer siRNA. sEVs were isolated from the pooled serum - each sample corresponds to the serum of 3 mice, mixed female and male (2:1) control mice (Adiponectin-Cre-Dicer fl/fl $^{\mathrm{f}}$ ) and aDicer-KO mice (10-16 weeks old) - by standard ultracentrifugation protocol and subjected to RNA isolation. qPCR revealed miR-20a-3p was detectable in 2 of the $3 \mathrm{aDicer}$-KO mice and none of the 5 control mice (Figure $3 \mathrm{~A})$. We have previously reported on reduced miR-186 and miR-324-5p in aDicer-KO mice (13). In addition, we have now performed silencing transfection of preadipocytes with Dicer siRNA. miR-20a-3p was significantly upregulated compared with control siRNA (Figure 3B). Herein, we show 3 models in which miR-20a$3 \mathrm{p}$ is upregulated, including in the circulation of humans with HIV lipodystrophy and reduced subcutaneous adipose Dicer expression, in human preadipocyte cell culture models with transfection of Dicer siRNA in knockdown studies, and in the circulation of a murine model with adipose Dicer knockout.

Gene expression signatures of adipocyte differentiation decreased by transfection of miR-20a-3p or the anti-mers of $m i R-186$ and miR-324-5p. Given these previous observations, we assessed whether the increased levels of sEV-carried miR-20a-3p or decreased levels of sEV-carried miR-186 and miR-324-5p might underlie the metabolic alterations observed in HIV lipodystrophy, specifically with respect to adipocyte dysregulation in the white adipose depot, including altered patterns of adipocyte differentiation capacity. To address this question, human preadipocytes were transfected with miR-20a-3p mimic, anti-miR-186, anti-miR-324-5p, or control nontargeting miRNA and then cultured in differentiation medium for 12 additional days to give rise to mature adipocytes, after which RNA was collected and subjected to RNA-Seq (Figure 4A). The transfection was performed in parallel with a fluorescence-labeled miRNA or a positive control miRNA targeting aldolase-A to confirm success. Analysis of the RNA-Seq data revealed 125 genes significantly upregulated, and 121 genes significantly downregulated in the mimic miR-20a-3p-treated cells, compared with miRNA control-treated cells (Figure 4B). Fifty-nine genes were significantly upregulated, and 203 genes were significantly downregulated 
A HIV/Non-Lipo vs. Non-HIV

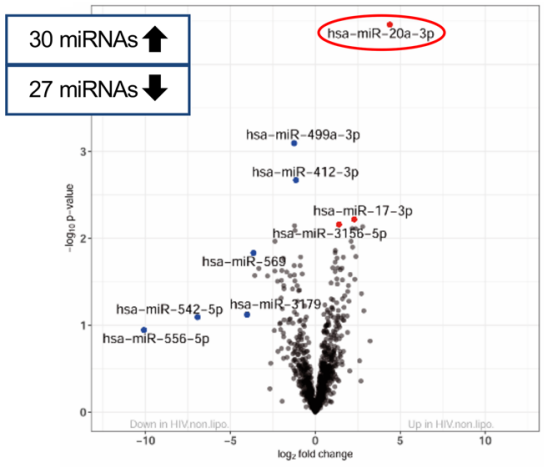

HIV/Lipo vs. HIV/Non-Lipo

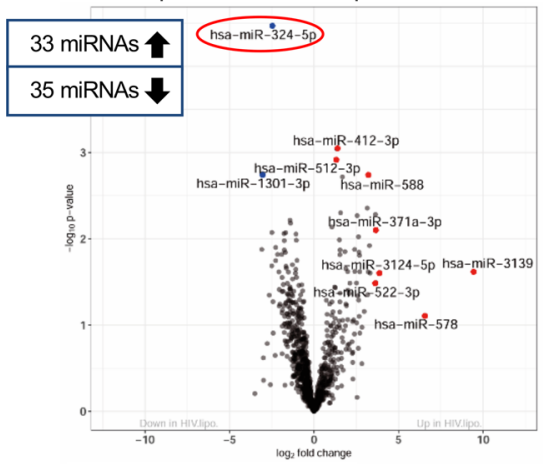

HIV/Lipo vs. Non-HIV

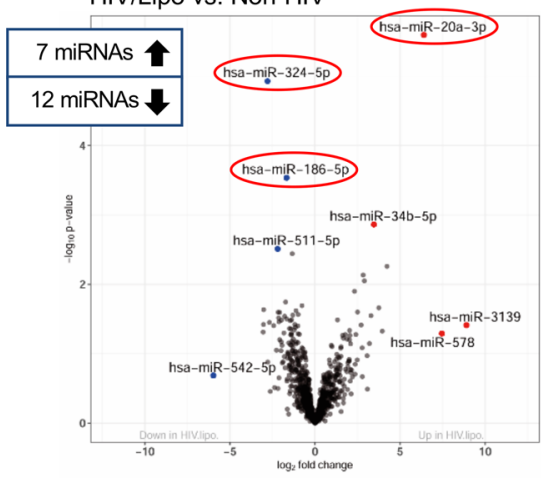

B

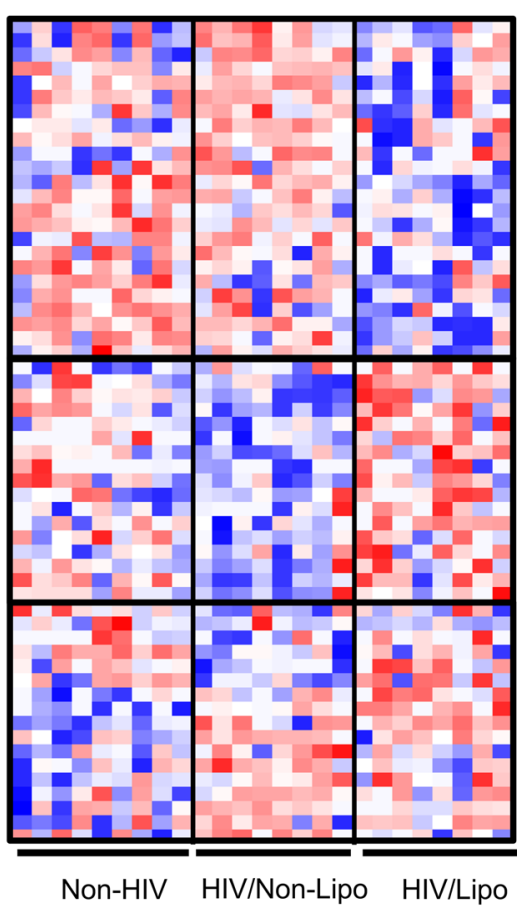

Color Key

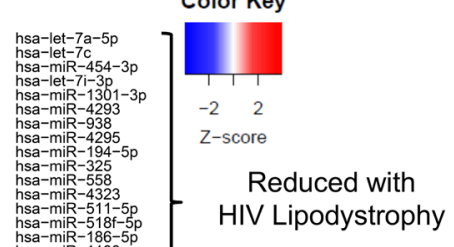

C

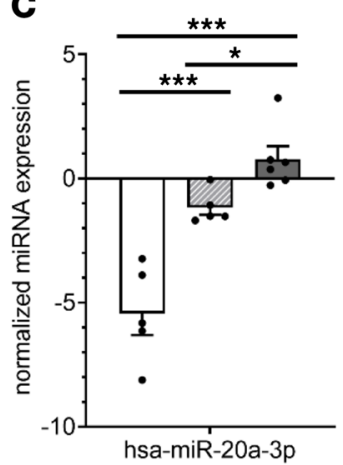

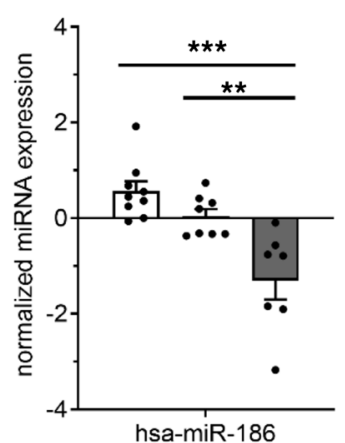

Figure 2. Circulating sEV miRNAs correlating to HIV infection and lipodystrophy. (A) Volcano plots representing the fold change and statistical significance for circulating sEV miRNAs for each of the group comparisons. The top regulated miRNAs are highlighted. The numbers in the rectangles indicate the number of upregulated and downregulated exosomal miRNAs $(P<0.05)$. The 3 miRNAs further studied (miR-20a-3p, miR-186, and miR-324-5p) are highlighted in a red circle. (B) Heatmap showing the abundance of the top regulated circulating sEV miRNAs shown in $\mathbf{A}$ in the conditions stated on the right side. High expression as indicated by normalized $\mathrm{Ct}$ is shown in red and low expression in blue. Each column represents an individual sample. (C) Normalized sEV miRNA expression obtained from miRNA profiling in each group for miR-20a-3p, miR-186, and miR-324-5p. Data are expressed as mean $\pm S E M$. ${ }^{*} P<0.05$, ${ }^{* *} P<0.01$, ${ }^{* *} P<0.001 . n=5-9$ per group applies to all the panels in this figure; statistical comparison in $\mathbf{A}-\mathbf{C}$ is assessed by Limma analysis.

when treating the cells with anti-miR-186 versus control group, and 64 genes were significantly upregulated and 203 miRNAs significantly downregulated when treating the cells with anti-miR-324-5p (Figure 4B). Surprisingly, both anti-miRs highly overlapped in their gene expression pattern while mimic miR-20a-3p resulted in a more distinct pattern (Figure 4C and Supplemental Figure 2, A and B). Among the pathways upregulated by mimic miR-20a-3p, we found several related to cellular adhesion and activity of the actin cytoskeleton (FAK-adhesion, ECM-receptor interaction, regulation of actin cytoskeleton) and others related to inflammatory processes (cytokine-cytokine receptor activation, hematopoietic cell lineage) (Supplemental Figure 2C). Only the proteoglycan pathway was slightly downregulated by mimic miR-20a-3p (Supplemental Figure 2C). In contrast, both anti-miRs upregulated pathways related to catabolism, such as oxidative phosphorylation, TCA cycle, or fatty acid degradation, and downregulated others related to cell adhesion and ligand pathways, such as Hippo and Wnt signaling pathways (Supplemental Figure 2D). 
Table 2. Correlations between sEV miRNAs among all participants

\begin{tabular}{|c|c|c|c|c|c|c|}
\hline & \multicolumn{2}{|c|}{ miR-20a-3p } & \multicolumn{2}{|c|}{$m i R-324-5 p$} & \multicolumn{2}{|c|}{ miR-186 } \\
\hline & $\rho$ & $P$ value & $\rho$ & $P$ value & $\rho$ & $P$ value \\
\hline miR-20a-3p & & & -0.71 & 0.002 & -0.74 & 0.002 \\
\hline miR-186 & & & & & & \\
\hline
\end{tabular}

To identify genes that might be coordinately regulated by the upregulation of circulating sEV-carried miR-20a-3p and the downregulation of miR-186 and miR-324-5p, we sought genes that would be regulated by all these 3 , the mimic miR-20a-3p, anti-miR-186, and anti-miR-324-5p, in the same direction. This analysis revealed that 7 genes were similarly upregulated and 47 genes were similarly downregulated by these treatments (Figure $4 \mathrm{~B}$ ). The commonly regulated genes by all treatments are shown in a heatmap (Figure 4C). The gene with the most significant downregulation by all 3 treatments was latent transforming growth factor beta binding protein 2 (Ltbp2) as shown by volcano plots (Figure 5A) and bar graphs (Figure 5B). In addition, other genes very significantly downregulated by all 3 treatments included nebulette ( $\mathrm{Nebl}$ ) and WNT1-inducible-signaling pathway protein 2 (Wisp2) (Figure 5, A and B). Ltbp2 is thought to relate to SREBP-1b and SREBP-1c and was shown to associate with BMI-adjusted waist circumference in GWAS (16). Interestingly, Nebl is a predicted target of all 3 miRNAs (miR-20a-3p, miR-324-5p, and miR-186) that was identified using several miRNA target prediction tools (TargetScan and Diana databases, target score $>85 \%$ ). Wisp2 participates in the induction of brown adipose tissue function, augmentation of insulin sensitivity, and regulation of preadipocyte commitment and PPAR $\gamma$ activation (17-19). Thus, our data suggest that the increased levels of circulating sEV-carried miR-20a-3p and decreased miR-186 and miR-324-5p associated with HIV lipodystrophy might influence adipocyte differentiation in several key ways, by affecting pathways related to lipid catabolism, inflammation, or cellular adhesion, among others, through effects on Ltbp2, Nebl, and Wisp2.

In addition, utilizing the publicly available National Center for Biotechnology Information Gene Expression Omnibus database GSE28073 comparing gene expression in HIV-infected patients (20), we found a reduction of $L t b p 2$ in both abdominal and dorsocervical adipose tissues from patients with HIV lipodystrophy compared with patients with HIV without lipodystrophy (Figure 6).

Assessment of adipocyte differentiation and inflammatory phenotype in knockdown studies of transfected human adipocytes. In order to determine the effects of the downregulation of Ltbp2, Nebl, and Wisp2 and their potential synergy in adipocyte differentiation, we performed silencing transfection of preadipocytes with either Ltbp 2 siRNA, Nebl siRNA, or Wisp2 siRNA individually, or a cocktail of all 3 siRNAs (containing one-third of each siRNA dose) 1 day prior to induction of differentiation. As a control, a nontargeting siRNA was used in parallel. RNA was collected at the end of the differentiation and subjected to RNA-Seq (Figure 7A). As shown in Supplemental Figure 3, single siRNA treatment efficiently downregulated ( $>80 \%$ reduction) the target genes. Triple siRNA combination efficiently downregulated Ltbp2 ( $\sim 80 \%$ reduction) but had a weaker but still significant reduction on $\mathrm{Nebl}(\sim 50 \%$ reduction) and had a very modest nonsignificant effect on Wisp2 ( $20 \%$ reduction) (Supplemental Figure 3$)$. As the triple siRNA combination had one-third of the dose of each siRNA, this differential targeting capacity for each target is likely due to lower efficiency at slightly lower doses.

We next analyzed how the downregulation of $L t b p 2, N e b l$, and Wisp 2 might affect processes involved in lipodystrophy, such as adipocyte differentiation. We found that knockdown of $L t b p 2$ significantly downregulated multiple gene markers of adipocyte differentiation, such as Fabp4, Ppary, C/ebpa, Fasn, adiponectin, Glut4, and CD36 (Figure 7B). Knocking down Wisp2 also led to a general decrease of several of these adipocyte differentiation markers, with effects on Fabp4, Ppar, Fasn, adiponectin, Glut4, and Srebp1c reaching statistical significance (Figure 7B). In contrast, Nebl siRNA did not lead to any significant changes. Combined knockdown of these targets using a combination of Ltbp2 siRNA, Nebl siRNA, and Wisp2 siRNA was additionally assessed in a supportive analysis and resulted in a significant downregulation of Fabp4, Ppary, Clebpa, Fasn, adiponectin, Glut4, and CD36 (Supplemental Figure 4B). Downregulation of genes related 
Table 3. Correlations between sEV miRNAs and metabolic parameters among all participants

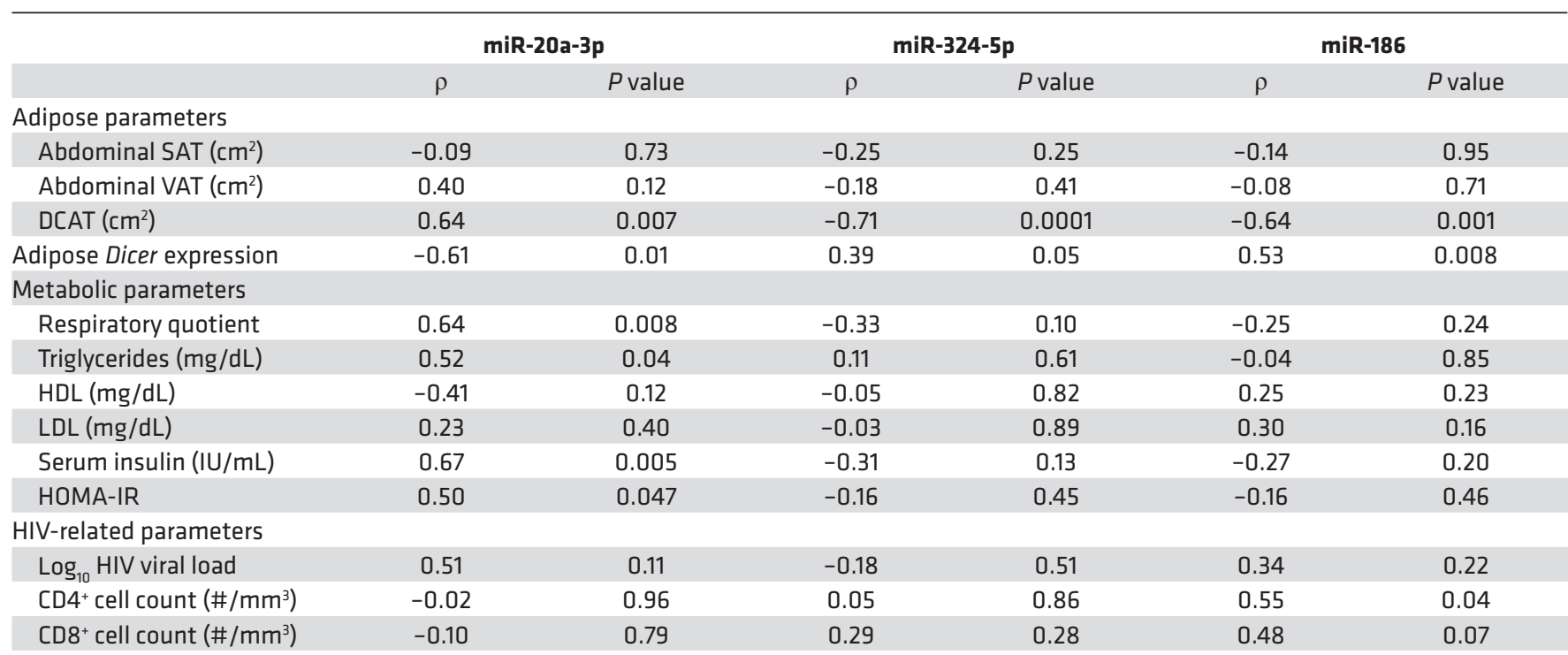

Relationships determined by Spearman's correlation coefficient.

to adipocyte differentiation were generally lower with $L t b p 2$ siRNA in contrast with the triple-combined siRNA (but at one-third of the concentration), suggesting the effect was driven predominantly by $L t b p 2 \mathrm{siR}$ NA. Mutations in lamins A and C have been associated with lipodystrophy and metabolic complications (21-23). In our studies, compared with the control siRNA, there was a significant downregulation of Lamin $C$ expression in both Ltbp2 siRNA and Wisp2 siRNA groups, while Lamin A expression did not appear to differ among all groups (Figure 7C and Supplemental Figure 4C). We also demonstrate decreased adipocyte differentiation in histological studies utilizing oil red $\mathrm{O}$ staining. Histologic images and quantitative data showed reduced oil red $\mathrm{O}$ staining after adipocytes were transfected with siRNA for Ltbp 2 and Wisp2 when compared with the control (Figure 8 and Supplemental Figure 5).

Another process intimately related to lipodystrophy is the activation of inflammatory pathways. Again, $L t b p 2$ knockdown had the greatest effects, leading to upregulation of $I L 1 b, I L 6$, and $C c l 20$, while the other treatments had no or very minor effects (Figure 7D and Supplemental Figure 4D). We saw consistent but nonsignificant trends toward increased expression of $I F N \beta$ in response to knockdown of $L t b p 2, N e b l$, and Wisp2. Thus, our data suggest that Ltbp2 is important for proper adipocyte differentiation and control of inflammation. The downregulation of Ltbp2 induced by elevated sEV-carried miR-20a-3p or decreased sEV miR-186 and miR-324-5p in patients with HIV lipodystrophy may underlie the impairment of adipose tissue development/differentiation and the increased activation of inflammatory processes seen in such patients and could be a potential target for future clinical studies (Figure 9).

No effects of knockdown of Ltbp2, Nebl, or Wisp2 were seen on markers of mitochondrial function, Tomm20 and $m t N D 2$. When assessing effects on markers of oxidative stress, no effects were seen on Catalase, Sod1, Sod2, or NADPHox-p40phos, but Hmox1 was decreased significantly in response to Ltbp2 (Supplemental Figure 6).

In exploratory analyses of effects on brown fat gene expression, we applied a beiging differentiation protocol to the preadipocytes. We observed nonsignificant decreases in brown adipose marker Ucp 1 with Ltbp2, Nebl, and Wisp2 siRNA compared with the control. In addition, Prdm16, a key beige adipose marker, demonstrated a relative decrease following transfection of $L t b p 2, \mathrm{Nebl}$, and Wisp 2 siRNA when compared with the control (Supplemental Figure 7).

\section{Discussion}

The HIV population frequently presents with a redistribution of fat and metabolic abnormalities, including dorsocervical adipose lipohypertrophy, ectopic fat accumulation, subcutaneous adipose dysfunction, insulin resistance, and dyslipidemia. These features closely resemble the phenotype of aDicer-KO mice (8), surmising a role for adipose-derived miRNAs in the pathogenesis of this syndrome. Indeed, downregulation 
A

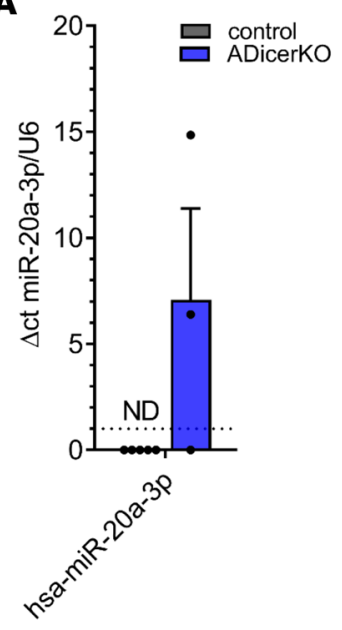

B

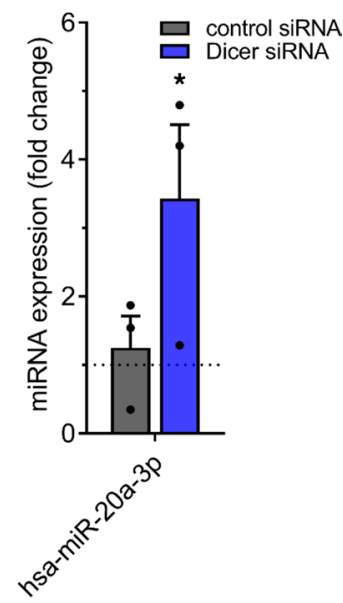

Figure 3. Expression of miR-20a-3p in alternative models of reduced Dicer expression. (A) Normalized sEV miRNA expression for miR-20a-3p in aDicer-KO mice versus control. (B) Normalized sEV miRNA expression for miR-20a-3p in adipocytes transfected with Dicer siRNA versus control. Data are expressed as mean \pm SEM. ${ }^{*} P<0.05 . n=5$ sera pools for control mouse group (each pool derives from sera of 3 mice), $n=3$ sera pools for aDicer-KO mouse group (each pool derives from sera of 3 mice), $n=3$ per group applies for $\mathbf{B}$. ND, not detected.

of Dicer expression in fat and subsequent dysregulation of adipose-derived miRNAs, many of which may be secreted in SEV and function as adipokines (13), could provide a novel mechanism for altered adipose function and related metabolic abnormalities in HIV lipodystrophy. Interestingly, HIV-related accessory proteins, for example viral protein $\mathrm{R}$ and trans-activator of transcription, have been reported to suppress Dicer expression $(6,24,25)$. While suppression of Dicer may have evolved as a mechanism to enhance infectivity of the HIV, there may be unintended metabolic consequences leading to a lipodystrophic phenotype.

To begin to investigate the role of Dicer in adipose and metabolic regulation in HIV, we previously assessed subcutaneous adipose expression of Dicer in a group with well-defined lipodystrophy and demonstrated reductions in adipose Dicer expression in association with key brown fat genes and clinical parameters (6). In the current study, we have performed sEV miRNA profiling in this well-phenotyped cohort of individuals with and without HIV in whom we have previously characterized adipose expression of Dicer. We hypothesized a unique miRNA signature among those with HIV lipodystrophy in relationship to dysregulation of Dicer. In these analyses evaluating sEV miRNA profiles, we identified 3 specific miRNAs (miR-20a-3p, -186, and -324-3p) as the top differentially regulated miRNAs using a stringent statistical approach. Notably, these miRNAs demonstrated a unique signature strongly related to Dicer expression and well-defined clinical lipodystrophy characteristics, including increased dorsocervical adipose tissue, as well as key metabolic and immune indices. In support of our findings, we were able to demonstrate upregulation of miR-20a-3p across 3 adipose-specific models of reduced Dicer expression: (a) in the circulation among patients with HIV lipodystrophy and reduced Dicer expression in the abdominal subcutaneous depot, (b) in the circulation of the aDicer-KO mouse, and (c) in a preadipocyte culture model transfected with Dicer siRNA. Follow-up experiments to manipulate these identified miRNA pathways in human cultured preadipocytes led to identification of potentially novel corresponding adipose gene targets of these miRNAs. Subsequent knockdown experiments of these genes through silencing transfection studies demonstrated key metabolic roles relevant to glucose and lipid metabolism.

We studied an HIV population who were well treated on ART, a group representative of the large population of aging individuals who are living with chronic HIV infection and demonstrate good immunologic control but nonetheless have an increased risk for mortality driven in part by metabolic complications. The HIV lipodystrophy cohort was identified based on the presence of dorsocervical lipohypertrophy on physical exam and confirmed based on quantitative measurements on MRI. Moreover, the cohort demonstrated reduced extremity fat via DXA, suggesting the common phenotype of loss of subcutaneous fat. Dicer expression was lowest in those with imaging-confirmed lipodystrophy, followed by HIV without lipodystrophy and then non-HIV controls (6). 
A

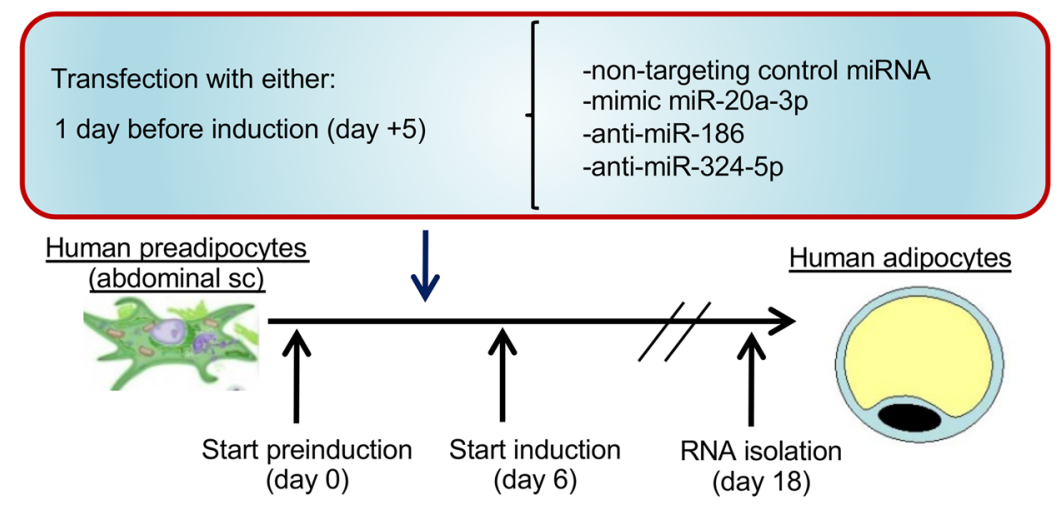

B

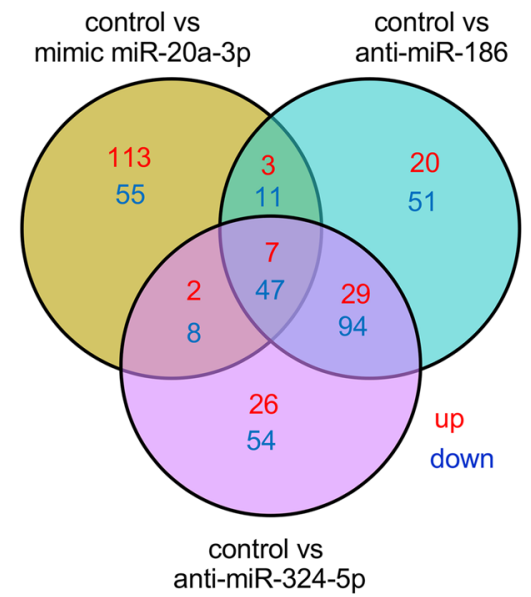

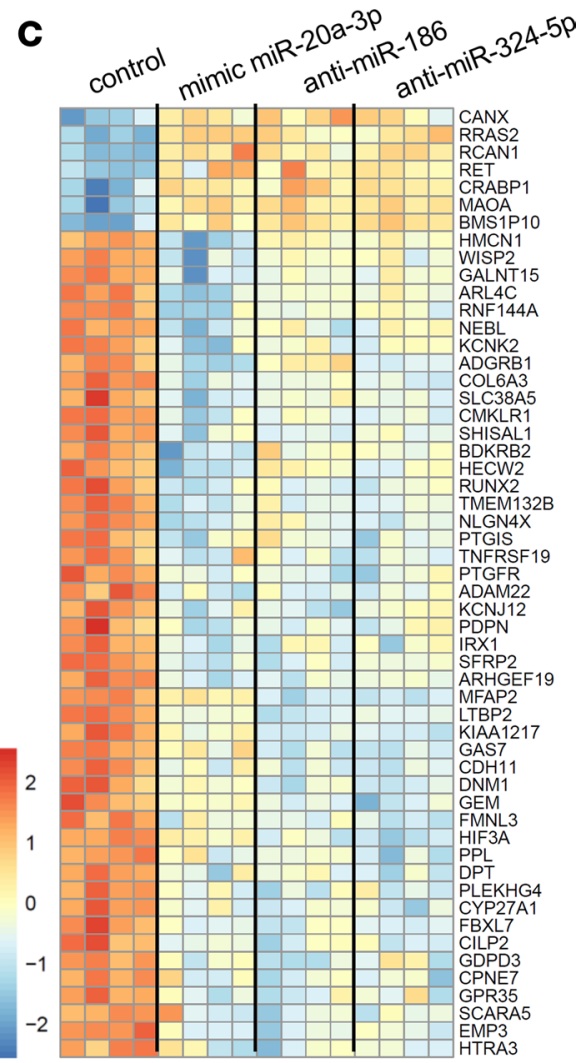

Figure 4. Overexpression of miR-20a-3p and inhibition of miR-186 and miR-324-5p alter transcriptome of differentiating adipocytes. (A) Diagram representing the experimental setup. (B) Venn diagram showing the number of genes upregulated (red) and downregulated (blue) for each comparison and the overlap among them. (C) Heatmap showing the commonly regulated genes by all treatments from the center of the Venn diagram in B. Red indicates high expression; blue indicates low expression. $n=4$ per group in all the panels; statistical comparisons in $\mathbf{B}$ and $\mathbf{C}$ assessed by Limma analysis.

We have previously shown the dorsocervical adipose tissue is a beige fat-like adipose depot that may develop as a compensatory mechanism to lipodystrophy (26). In a prior study we showed that reduced Dicer expression in subcutaneous adipose tissue is related inversely to dorsocervical adipose tissue accumulation. We now demonstrate that increased dorsocervical adipose tissue is strongly correlated to the miRNA signature we have identified, such that greater dorsocervical adipose tissue is associated with upregulation of miR-20a-3p and downregulation of miR-186 and miR-324-5p. Moreover, in exploratory analyses, we observed potential effects of key dysregulated genes in this study, Ltbp2, Nebl, and Wisp2, on 2 genes involved in browning of white adipocytes, Ucp 1 and Prdm16. Further studies should assess the effects of dicer-related downstream effect on brown and beige fat pathways in specific adipose depots.

In our study, pathway analyses revealed that highly relevant clusters important to metabolism and cytokine signaling were significantly affected by miR-20a-3p mimic as well as anti-miR-186 and antimiR-324. These additional analyses provide supporting evidence that the 3 top differentially regulated miRNAs identified in this study are linked to adipocyte biology and physiologic systems applicable to HIV lipodystrophy. In addition, there may be clinical utility to evaluating whether these $3 \mathrm{sEV}$ miRNAs could serve as a diagnostic tool using a simple, noninvasive technique to identify a clinical presentation of HIV lipodystrophy, for which objective measures may be lacking. Moreover, we saw that upregulation of miR-20a-3p related to greater triglycerides and insulin resistance, further suggesting a link to the clinical lipodystrophy phenotype. Further studies may identify other miRNAs potentially important in mediating metabolic effects in relationship to reduced Dicer in HIV. 
A

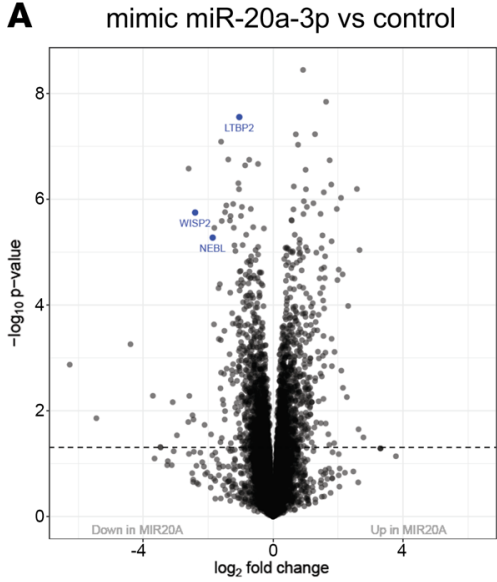

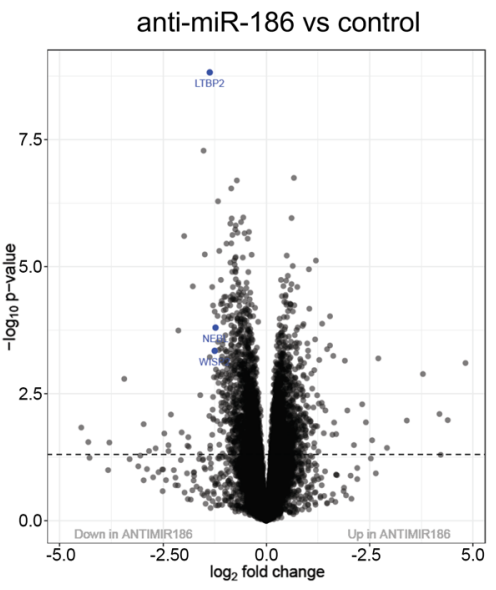

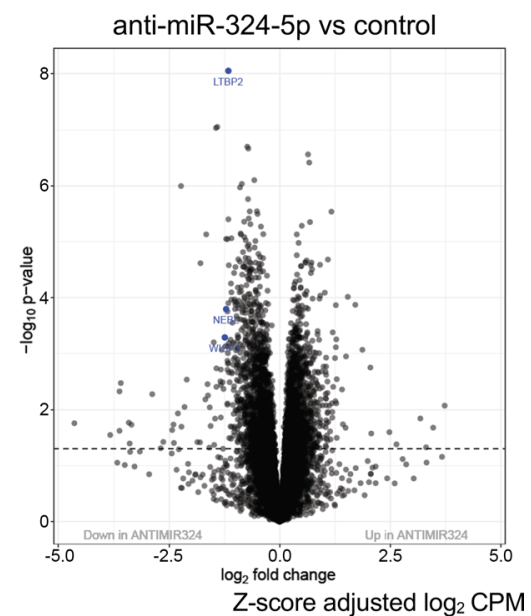

B
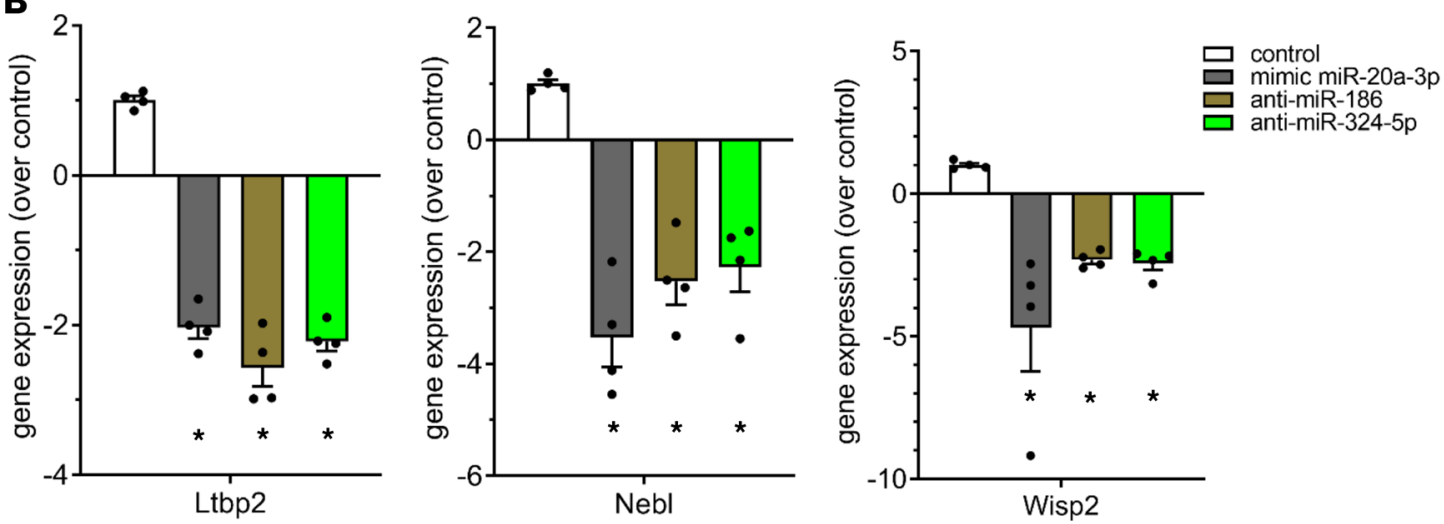

Figure 5. Overexpression of miR-20a-3p and inhibition of miR-186 and miR-324-5p of differentiating adipocytes demonstrates downregulation of Ltbp2, Nebl, and Wisp2. (A) Volcano plots illustrating the fold change and statistical significance for the profile of each comparison. The dashed line in each plot indicates $P$ value 0.05 . The genes that are the subject of further study (Ltbp2, Nebl, and Wisp2) are highlighted in blue. (B) Bar graphs showing the degree of downregulation of Ltbp2 (left), Nebl (middle), and Wisp2 (right graph) induced by each of the treatments. Data are expressed as mean \pm SEM. ${ }^{*} P<0.05 . n=4$ per group in all the panels; statistical comparisons in $\mathbf{A}$ and $\mathbf{B}$ assessed by Limma analysis.

There are limited adipose and metabolic data about the roles of the 3 top differentially regulated miRNAs identified in this study in other cellular and disease models. A recent study of Mycobacterium tuberculosis suggested that overexpression of miR-20a-3p contributed to mycobacterial survival in a macrophage cell line and may aid in the host immune response (27). In this way, changes in miR-20a-3p and other miRNAs could help enhance survival of HIV in the host and have a secondary consequence of metabolic complications. In addition, miR20a-3p has also been implicated in the MAPK signaling pathway and phosphatidylinositol signaling system (28). Consistent with the current study, miRNA-20a-3p has been shown to be elevated among patients with type 2 diabetes mellitus compared with healthy individuals when isolated from circulating plasma ectosomes (29). A recent study showed that miR-186 is upregulated in a T lymphoblast cell line following acute HIV infection, and this is associated with reduced Dicer expression in these cells (30). In our study, we have shown, in contrast, reduced miR-186 in circulating SEVs from chronically treated HIV-infected patients on ART, demonstrating reduced Dicer expression in the adipose depot. With regard to miR-324-5p, which we show to be downregulated in HIV lipodystrophy, this miRNA has also been shown to be downregulated in circulating sEVs of patients with type 2 diabetes mellitus while demonstrating an inhibitory effect on diabetes-related inflammation (31). miR324-5p overexpression has been linked to enhanced glucose uptake in oxygen glucose-deprived neurons (32) and has been reported to promote Wnt signaling (33). Consistent with this, we found downregulated Wnt signaling in adipocytes following treatment with anti-miR-324-5p.

In the context of HIV lipodystrophy, few other miRNAs have been reported to have a potential role. One study demonstrated that miR-218 was upregulated in the subcutaneous adipose of patients with HIV lipodystrophy, and this was associated with reduced lipin1 levels, a putative target of this miRNA. 


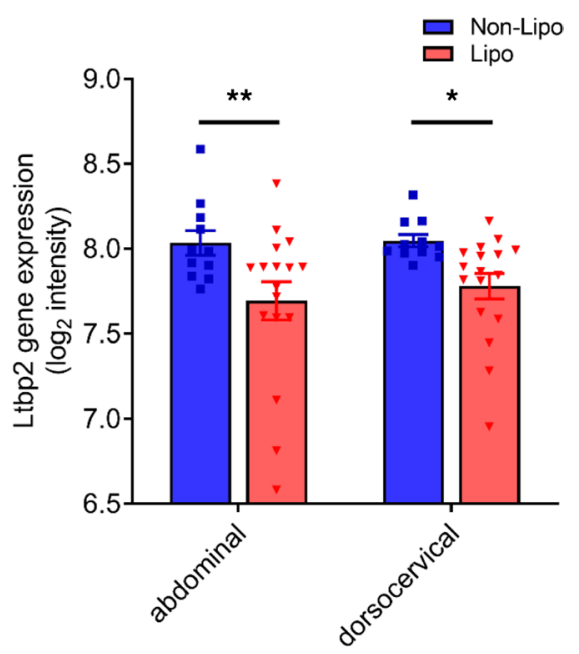

Figure 6. Ltbp2 expression is downregulated in another cohort of HIV-infected patients with lipodystrophy. Ltbp2 gene expression in the abdominal subcutaneous and dorsocervical adipose tissue depots from a cohort of HIV-infected patients without or with lipodystrophy. $n=11$ for nonlipodystrophic patients, $n=17$ for lipodystrophic patients. Data are expressed as mean \pm SEM. ${ }^{*} P<0.05,{ }^{* *} P<0.01$; statistical comparisons were assessed by Limma analysis.

Furthermore, cotreatment of 3T3-L1 cells with a miR-218 mimic and lopinavir/ritonavir decreased Glut4 levels $(34,35)$. This prior study also reported that 21 miRNAs out of 754 were overexpressed by 2 -fold in the subcutaneous adipose among 8 individuals with HIV lipodystrophy compared with 8 uninfected individuals. Interestingly, in contrast to our findings in sEV miRNAs, the authors found that miR-186 was upregulated in the adipose depot but did not report any data on miR-20a-3p or miR-324-5p in the adipose tissue or on altered pattern of circulating miRNA.

We have previously reported significant miRNAs affected in the aDicer-KO model (8) and now show general concordance between findings in these mouse and human studies, for example, in the relative increase in miR-20a-3p and reductions in reduced miR-186 and miR-324-5p. Some differences between studies could be due to selective sorting of certain miRNAs into sEVs $(36,37)$, differing biology in the mouse versus human, or differences in the mechanism of Dicer dysregulation in these models, e.g., adipose-specific total genetic knockout of Dicer in the mouse study (8) versus physiological regulation in multiple tissues by HIV-related factors in the current study. Taken together, these data from multiple experiments, including the observation of increased miR-20a-3p expression in preadipocytes in response to Dicer siRNA, suggest an effect of reduced dicer to increase miR-20a-3p and affect other miRNAs.

One interesting aspect of these studies was the finding that $L t b p 2$ transcription was significantly downregulated in human preadipocyte cultures by all 3 miRNAs observed to be altered in HIV lipodystrophy. Similarly, expression of Nebl and Wisp2 was also decreased by all 3 miRNAs. Furthermore, we showed that Ltbp2 was reduced in both abdominal and dorsocervical adipose tissue biopsies from another cohort of patients with HIV who developed lipodystrophy when compared with adipose tissue from patients with HIV who did not develop lipodystrophy (20), further indicating a critical role of Ltbp2 in HIV lipodystrophy. Moreover, in the present study, we demonstrated that downregulation of Ltbp2 in adipocytes by siRNA affected key regulators of adipocyte differentiation, metabolic regulation, cellular structure, and inflammation. Indeed, knockdown of $L t b p 2$ in human cell culture of preadipocytes consistently resulted in downregulation of several key genes associated with maturation of adipocytes, including C/ebpa and CD36, highlighting the role of $L t b p 2$ as a potential mechanistic factor contributing to subcutaneous adipose dysfunction and related metabolic abnormalities in HIV lipodystrophy. To complement these changes in gene expression, we also found reduced oil red $\mathrm{O}$ expression in adipocytes transfected with Ltbp2. Of note, prior work in HIV has shown adipose dysfunction among individuals with HIV, with lower mRNA concentrations of key adipogenic differentiation factors, including $C / e b p \alpha$ and $\beta, P p a r \gamma$, and Srebp1c (38). This prior work also demonstrated Srebp1c mRNA concentrations correlated negatively with inflammatory cytokine levels (38).

Several genes downregulated by knockdown of $L t b p 2$ are also linked to fatty acid oxidation and lipid metabolism pathways, such as Fasn, Fabp4, and adiponectin. Impaired fatty oxidation is a characteristic 
A

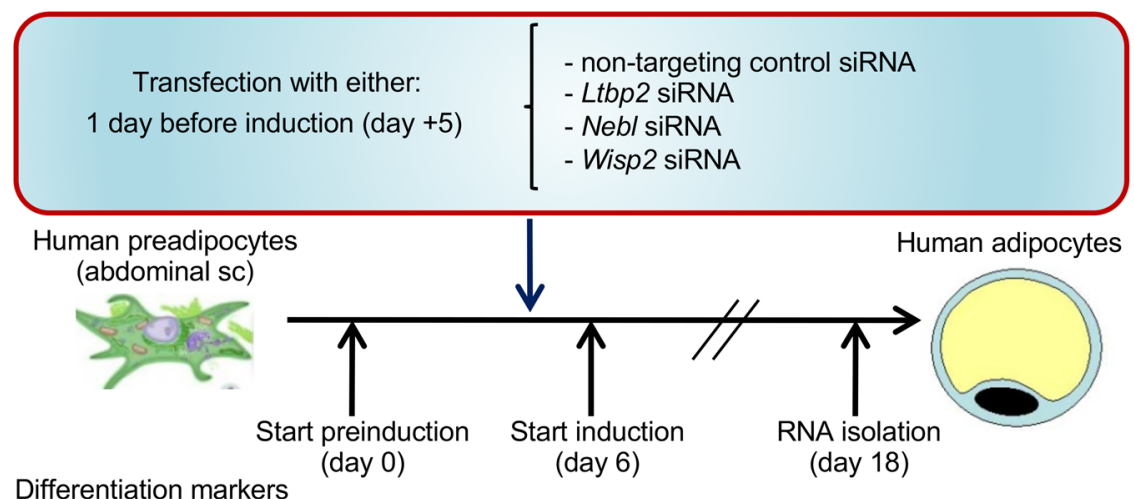

B Differentiation markers (day 0) (day 6)

(day 18)
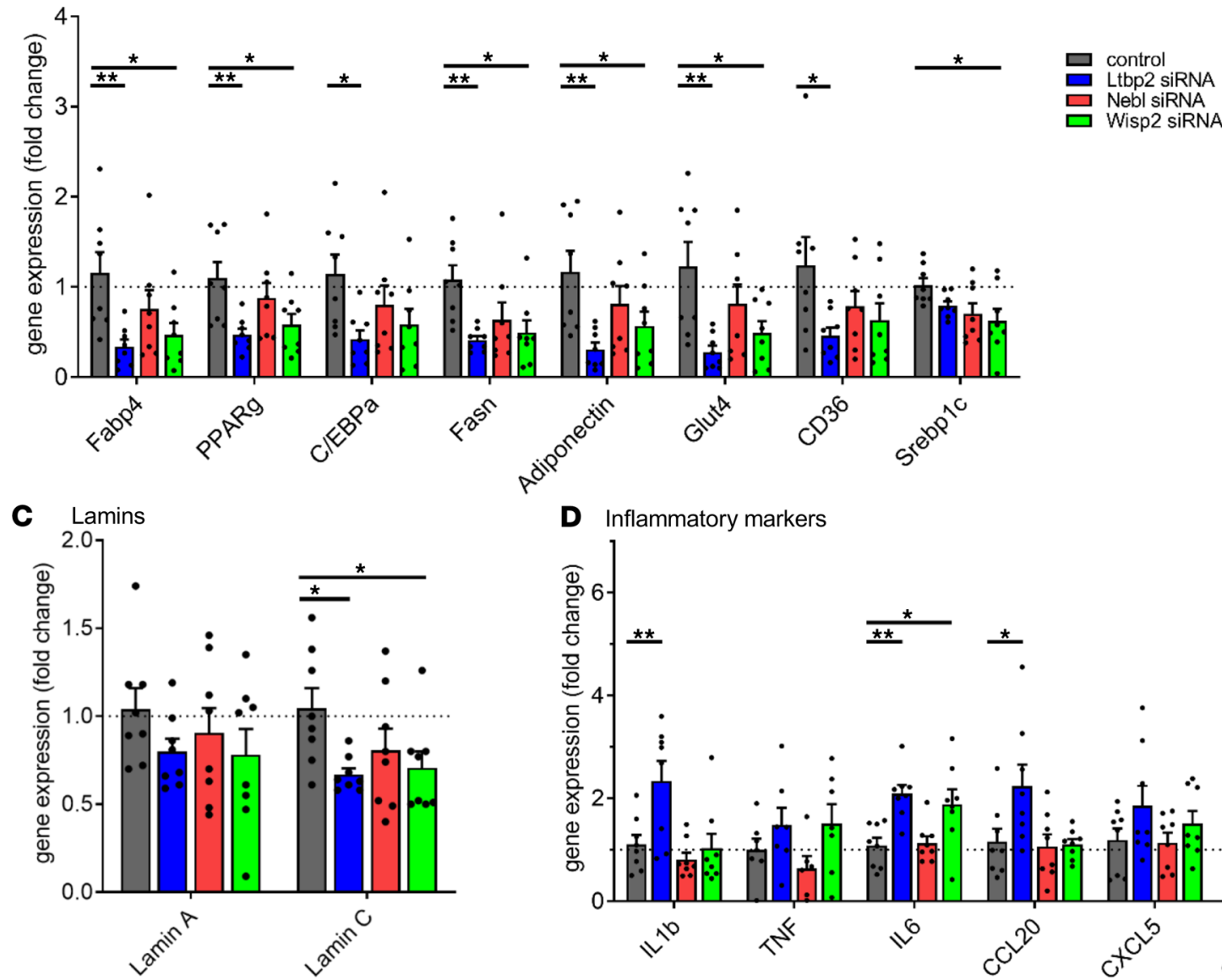

Figure 7. Ltbp2 regulates adipocyte differentiation, Lamin C expression, and inflammation. (A) Diagram representing the experimental setup. (B) Effect of the treatment of preadipocytes with negative control, Ltbp2, Nebl, and Wisp2 siRNA on the indicated differentiation markers. (C) Effects of the indicated treatments on the expression of Lamin A and Lamin C. (D) Effects of the indicated treatments on the expression of the inflammatory markers shown below. Data are expressed as mean \pm SEM. ${ }^{*} P<0.05,{ }^{* *} P<0.01 . n=8$ in all panels; statistical comparisons in B-D were performed by 1 -way ANOVA followed by Dunnett's test comparing individual groups to control.

of HIV lipodystrophy $(39,40)$. Adiponectin, Glut4, and Ppary, integral genes to insulin action and sensitivity, were also dampened by Ltbp2 downregulation. Insulin resistance is a common clinical finding in HIV lipodystrophy often presenting in association with other metabolic sequelae, such as ectopic fat accumulation and inflammation $(10,12,41)$. While existing miRNA databases do not report Ltbp2 as a target of our identified miRNAs, our data reveal a clear link between these miRNAs and Ltbp2. Knockdown of Wisp2 also resulted in downregulation of markers of adipocyte differentiation though to a somewhat lesser degree than Ltbp2. In that regard, combined loss of $L t b p 2, N e b l$, and Wisp2 did not have an additive effect across critical metabolic genes above that of $L t b p 2$, suggesting that these genes act in the same pathway. 

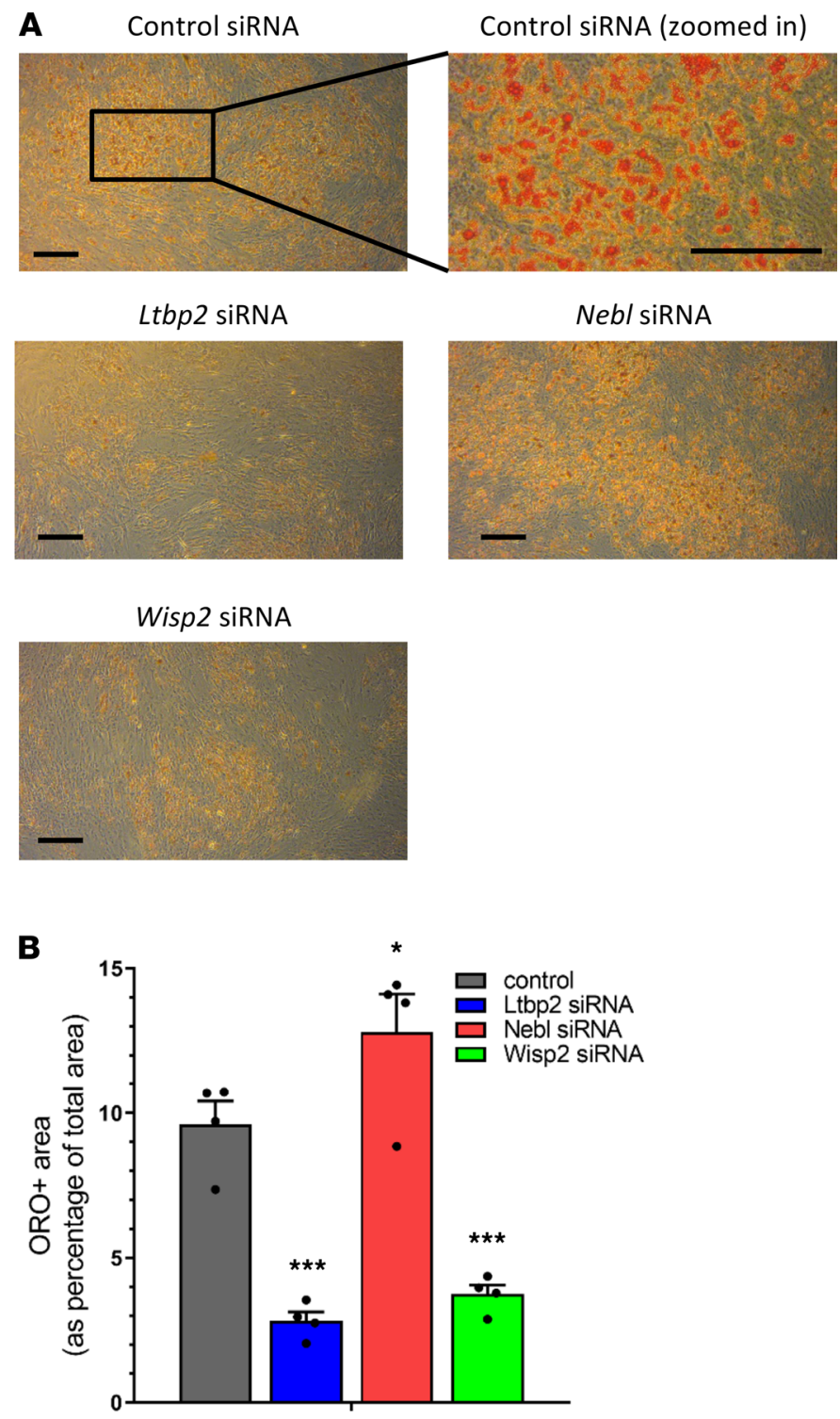

Figure 8. Knockdown of Ltbp2 demonstrates reduced adipocyte differentiation. (A) Histologic images of adipocytes treated with $L t b p 2, \mathrm{Nebl}$, and Wisp2 siRNA stained with oil red O. (B) Quantitative measure of oil red $\mathrm{O}$ staining as a percentage of total area. Scale bar: $100 \mu \mathrm{m} . n=4$, data are expressed as mean $\pm \mathrm{SEM}$. ${ }^{*} P<0.05,{ }^{* * *} P<0.001$; statistical comparisons was performed by 1-way ANOVA followed by Dunnett's test comparing individual groups to control.

Mutations in Lmna and Ppary are well recognized in familial partial lipodystrophies and therefore could play a role in this acquired form of lipodystrophy. In this study, we determined that knockdown of $L t b p 2$ and Wisp 2 resulted in significant downregulation of Lamin C. The Lmna gene encodes both lamins A and C isoforms generated by alternative splicing of Lmna. Lmna gene mutations generally disrupt both lamin A and lamin C in partial lipodystrophy (42). Missense mutations on the Lmna gene, predominantly identified on exon 8 , have been reported to cause a lipodystrophic phenotype $(43,44)$. There are reports of lamin A-specific mutations in partial lipodystrophy (43) and lamin C-specific mutations in generalized lipodystrophy (42). Interestingly, protease inhibitors have been linked to altered lamin C (45). We demonstrate reduced Lamin C in response to knockdown of key genes dysregulated by miRNAs in HIV lipodystrophy in cultured human preadipocytes. This occurs in vitro, independent of any ART exposure, but in vivo, it is possible that ART use may further compound this effect on Lamin $C$. The altered expression of these genes regulated by $L t b p 2$ expression may contribute to fat dysregulation and subsequent metabolic complications in HIV lipodystrophy.

Finally, 3 inflammatory markers, $I L 1 \beta, I L 6$, and $C c L 20$, were increased with knockdown of $L t b p 2$. HIV lipodystrophy is associated with an inflammatory phenotype; i.e., subcutaneous adipose tissue shows an increased 


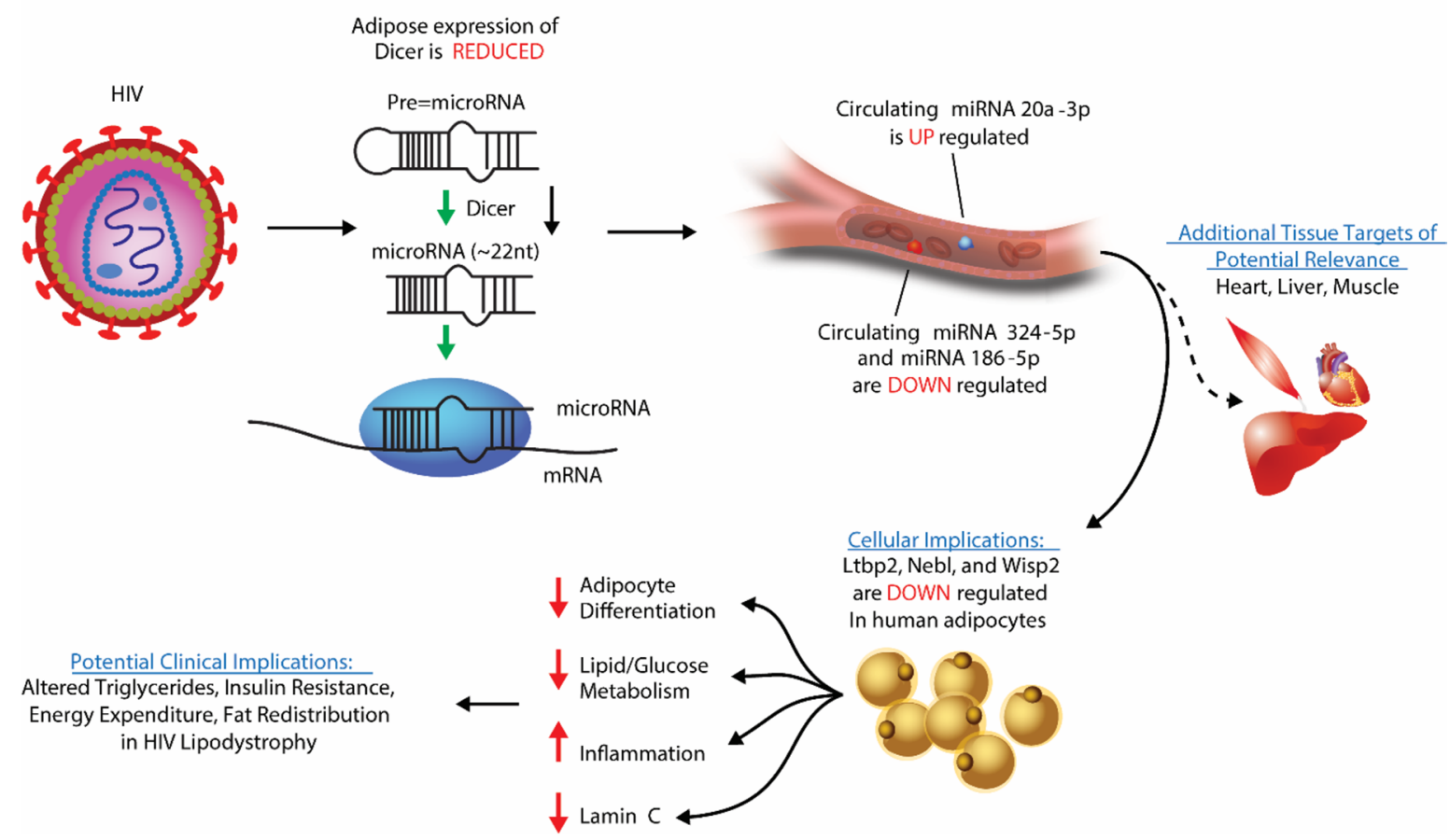

Figure 9. Hypothesized translational model of adipose dysfunction and metabolic complications in HIV lipodystrophy. Accessory proteins of the HIV may suppress Dicer expression in the adipose tissue. Lack of Dicer in adipose tissue leads to lipodystrophic changes in fat redistribution, metabolic dysregulation, and alteration of the levels of circulating exosomal miRNAs; i.e., sEV-carried miR-20a-3p is upregulated, and miR-324-5p and miR-186 are downregulated in serum taken from lipodystrophic patients with HIV. These sEVs would target preadipocytes, inducing downregulation of $L t b p 2$, $\mathrm{Nebl}$, and Wisp2, which would subsequently impair adipocyte differentiation, inflammation, and Lamin C expression. Thus, reduced dicer expression in the adipose among HIV-infected patients may contribute to an altered miRNA signature and adipocyte differentiation and inflammation, which could have potential clinical implications relevant to HIV lipodystrophy. Other tissues affected in HIV lipodystrophy, such as the heart, liver, and muscle, could also be relevant targets of this pathway.

infiltration with inflammatory cells. Importantly, IL-6, and to a lesser extent IL-1 $\beta$, have been linked to HIV lipodystrophy (46-48). Of note, knockdown of Ltbp2 was also associated with reduced Hmox1. Knockdown of Hmox1 could have an inflammatory effect as HMOX1 catalyzation can result in antiinflammatory responses by upregulation of interleukin 10 and interleukin 1 receptor antagonist expression $(49,50)$. Our data suggest altered miRNAs regulate $L t b p 2$, which in turn may increase inflammatory gene expression in adipose tissue, thus providing an additional mechanism for this effect. Other inflammatory markers, including IFN $\beta$, were generally increased but did not reach significance with knockdown of Ltbp2. Dicer has recently been identified to repress interferons and the antiviral response in other cell types (51); thus, our data showing a trend toward increased interferon with reduced Dicer show a potential consistent effect in adipocytes.

This study has a number of strengths and some limitations. A strength of our study is the translational exploration of a clinical phenotype. Leveraging our preclinical model of the aDicer-KO mouse, we evaluated the implications of reduced adipose Dicer expression in HIV lipodystrophy on sEV-carried miRNAs and further tested manipulation of these miRNAs, leveraging RNA-Seq to arrive at key genes contributing to critical adipogenic and inflammatory pathways. We were unable to collect additional subcutaneous adipose tissue samples in our human subjects to complement our RNA-Seq studies conducted using cultured human preadipocytes, but we validated the potential role of $L t b p 2$ in a relevant HIV database of adipose gene expression (20). Our data profiling circulating sEV patterns do not allow us to discern whether reduced Dicer in subcutaneous adipose is directly affecting local miRNAs, and whether such altered miRNAs affect local adipogenic pathways in a paracrine fashion, distant adipose tissues, or other organs. However, our adipocyte transfection study using Dicer siRNA suggests the adipocyte may be directly involved in a paracrine-like effect. Future studies should now investigate this discovered miRNA signature and related target genes to determine effects on key adipogenic pathways in critical adipose depots, including browning of white adipose tissue and oxidative stress, as well as metabolic pathways in other critical organs, such as 
the liver, to determine how this mechanism contributes to overall metabolic dysregulation in HIV. Future studies should also assess if these pathways are important in other conditions of reduced Dicer, such as aging and overfeeding $(9,52)$, which are characterized by metabolic dysregulation.

In the current investigation, we were unable to disentangle any specific contribution of ART in reducing Dicer and dysregulating circulating miRNAs. Nonetheless, our experiments in adipocyte cultures were performed in the absence of any ART, suggesting that the downstream consequences of reduced Dicer and altered miRNAs on adipose function occur independently of ART. Further studies should investigate this question.

In summary, we conclude miR-20a-3p functions as a key marker among individuals with HIV lipodystrophy with altered adipose tissue Dicer expression. Overexpression of miR-20a-3p was shown to promote metabolic perturbations, potentially through the downregulation of $L t b p 2$ in the adipocyte, leading to dysregulation in critical markers of adipose differentiation and inflammation, and a relevant lipodystrophy gene, Lamin $C$. As such, these studies provide a plausible mechanistic schema, whereby miR-20a-3p downregulates $L t b p 2$, leading to adipocyte dysfunction in HIV lipodystrophy. This study demonstrates a potentially novel pathway that can be targeted to improve adipose dysfunction in this population.

\section{Methods}

Study participants. Eighteen participants with HIV, 9 with and 9 without lipodystrophy, and 9 uninfected participants without HIV were previously recruited in an observational study to assess Dicer expression in the abdominal subcutaneous adipose tissue (6). All participants were male between the ages of 18 and 60 years with a BMI $18-35.0 \mathrm{~kg} / \mathrm{m}^{2}$. Among participants with HIV, ART use had been stable for more than 12 months. A single investigator assessed for HIV-related lipodystrophic changes in fat. Lipodystrophy was characterized by the presence of excess dorsocervical fat accumulation. MRI also confirmed the examination (see MRI methods below). Major exclusion criteria included hemoglobin less than $10.0 \mathrm{~g} / \mathrm{dL}$; known history of diabetes and use of antidiabetic medications; abnormal thyroid function; use of glucocorticoids, growth hormone, growth hormone-releasing hormone, or other anabolic therapies within 3 months of enrollment; current substance abuse; or active or serious chronic medical conditions other than HIV.

Assessment of fat redistribution. MRI of the neck was performed on a Siemens 3T Trio magnetic resonance system using phased-array neck and body matrix coils. A volumetric 3D Dixon gradient-echo multiecho pulse sequence with 6 echo times (repetition time $=20 \mathrm{~ms}$; echo time $=2.46,6.15,9.84,12.3,14.76,17.22$ $\mathrm{ms}$; flip angle $=5$; slice thickness $=3 \mathrm{~mm}$; field of view $=42 \mathrm{~cm}$; matrix $=256 \times 256$ ) was employed. Sagittal images were reconstructed to identify the level of C7 vertebral body. Axial images at the level of C7 were reconstructed and used for measurement of dorsocervical adipose tissue area. Vertical reference lines were placed along the lateral border of the vertebral body of $\mathrm{C} 7$ and projected over dorsocervical adipose tissue, providing standardized lateral boundaries for the fat depot. Dorsocervical adipose tissue was demarcated anteriorly by the paraspinal muscles and posteriorly by the dorsocervical skin. Area measurements within these boundaries were expressed in square centimeters. In addition, MRI was acquired using an axial T1-weighted, fat-suppressed pulse sequence obtained at the level of L4 vertebral body for determination of visceral and subcutaneous fat areas utilizing commercial software (Vitrak, Merge e/Film).

Biochemical and metabolic assessment. Participants presented after an overnight fast for blood sampling. $\mathrm{CD}^{+}$and $\mathrm{CD}^{+} \mathrm{T}$ cell counts were assessed by flow cytometry. HIV viral load was determined by ultrasensitive real-time PCR (Roche cobas amplicor). As previously described, Dicer expression was determined from subcutaneous adipose tissue biopsies (6).

Abdominal fat biopsy. Surgical sampling of subcutaneous abdominal fat was performed under local anesthesia with $1 \%$ lidocaine, using a $4 \mathrm{~mm}$ diameter punch biopsy. Specimens were snap-frozen in a dry ice/ ethanol bath and immediately transferred to $-80^{\circ} \mathrm{C}$.

Animals. Generation of adipocyte-specific Dicer-KO mice (aDicer-KO; Adiponectin-Cre+Dicer ${ }^{\mathrm{fl} / \mathrm{fl}}$ ) and control mice (Adiponectin-Cre-Dicer ${ }^{\mathrm{f} / \mathrm{fl}}$ ) was described elsewhere (8). Female and male mice aged between 10 and 18 weeks were used for the experiments. Mice were anesthetized with Avertin (MilliporeSigma), and blood was collected by cardiac puncture.

Human preadipocytes and white adipogenesis differentiation. Human white preadipocytes were provided by the adipocyte core of the Boston Nutrition Obesity Research Center (BNORC). Abdominal subcutaneous fat tissue was obtained from people undergoing plastic surgery. Donors were 30-60 years old, female, of BMI range $21.7-45.7 \mathrm{~kg} / \mathrm{m}^{2}$, and nondiabetic. Adipose stromal cells were isolated as previously described (14). Briefly, minced tissue was treated with collagenase solution ( $1 \mathrm{mg} / \mathrm{mL}$ HBSS) (type 1 , Worthington Biochemical) in 
a continuous shaker at $37^{\circ} \mathrm{C}$ for 2 hours. The digested tissue was filtered through a $250 \mu \mathrm{m}$ mesh (Component Supply, Inc.). Cells were centrifuged at $500 \mathrm{~g}$ for 10 minutes at room temperature. The red blood cells in the cell pellets were lysed $\left(0.154 \mathrm{mM} \mathrm{NH}_{4} \mathrm{Cl}, 10 \mathrm{mM} \mathrm{K}_{2} \mathrm{HPO}_{4}\right.$, and $0.1 \mathrm{mM}$ EDTA, pH 7.3). The washed cells were plated using $\alpha$-MEM (Gibco, Thermo Fisher Scientific) with 10\% FBS (Gemini Bio Products) and 100 U/ $\mathrm{mL}$ penicillin with $10 \mu \mathrm{g} / \mathrm{mL}$ streptomycin (pen/strep) (Corning). For the experiments, cells were grown in DMEM high glucose with $20 \%$ FBS and $1 \%$ pen/strep until confluence. At day 0 of the differentiation proto-

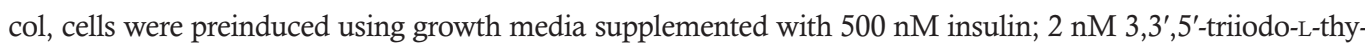
ronine (T3); and $1 \mu \mathrm{M}$ rosiglitazone. This medium was renewed every $2-3$ days. At day 6 , cell differentiation was induced using growth medium supplemented with $500 \mathrm{nM}$ insulin, $2 \mathrm{nM}$ T3, $1 \mu \mathrm{M}$ rosiglitazone, 0.54 mM 3-isobutyl-1-methylxanthine (IBMX), $33 \mu \mathrm{M}$ biotin, $17 \mu \mathrm{M}$ pantothenate, $0.1 \mu \mathrm{M}$ dexamethasone, and 30 $\mu \mathrm{M}$ indomethacin. This medium was renewed every 2-3 days for 12 additional days, after which cells can be considered fully differentiated (day 18 of differentiation protocol). All chemicals and reagents were purchased from MilliporeSigma unless otherwise noted. The mimic and anti-miR transfection, with $83 \mathrm{nM}$ mimic miR20a-3p (Horizon Discovery, Dharmacon) and anti-miR-186 and anti-miR-324-5p (miRVana, Thermo Fisher Scientific) mixed with lipofectamine RNAiMAX (Thermo Fisher Scientific), was done at day 5 after starting the preinduction phase. As controls, a positive control miRIDIAN microRNA Mimic targeting aldolase-A and a miRIDIAN Dy-547-labeled miRNA Transfection Control (both from Horizon Discovery, Dharmacon) were transfected in parallel. For the transfection with siRNAs against Ltbp2, Nebl, Wisp2, or nontargeting control siRNA, we used $16.67 \mathrm{nM}$ each single siRNA in combination with lipofectamine RNAiMAX. For the triple siRNA combination, one-third of each siRNA of the above siRNAs was used (5.55 nM each). Transfection with Dicer siRNA (Horizon Discovery, Dharmacon) was also performed at day 5 after starting the preinduction phase. One day later differentiation was induced as described above, and samples were collected for RNA isolation 6 days after differentiation induction.

Human preadipocytes and beige adipogenesis differentiation. Human white preadipocytes were obtained and grown until confluence as described in the previous section. After reaching confluence, cells were transfected with siRNAs against Ltbp2, Nebl, or Wisp2 or the triple combination as done for white adipogenesis above. One day after transfection with siRNAs, beige adipogenesis was induced based on the protocol described by Singh et al. (53), with slight modifications. Briefly, confluent cells were induced to beige differentiation by adding the following reagents resuspended in DMEM/F12 (Thermo Fisher Scientific, 11965118) with 10\% FBS and 1\% pen/strep: $200 \mathrm{ng} / \mathrm{mL}$ recombinant hIGF-I (Peprotech, 100-11), $8 \mathrm{ng} / \mathrm{mL}$ human basic FGF (Miltenyi Biotech, 130-093-838), 100 ng/mL human BMP7 (R\&D Systems, 354-BP), $10 \mu \mathrm{M}$ Y27632 (Cayman Chemical, 10005583), $2 \mu \mathrm{M}$ rosiglitazone (MilliporeSigma, R2408), $1 \mathrm{nM}$ T3 (MilliporeSigma, T6397), $1 \mu \mathrm{M}$ dexamethasone (MilliporeSigma, D4902), and $500 \mu \mathrm{M}$ IBMX (MilliporeSigma, I5879). Medium was replaced every 3 days. Lipid droplet accumulation was readily observable a few days after induction and increased with time until reaching approximately $80 \%-90 \%$ of the plate at day 21 after induction. On this day, cells were washed with PBS and added to TRIzol for RNA isolation as described below.

$s E V$ isolation. $\mathrm{sEV}$ isolation was performed using standard differential ultracentrifugation protocol (15) on 2-3 mL of serum from each of the 27 participants ( $9 \mathrm{HIV} /$ lipo, $9 \mathrm{HIV} /$ nonlipo, 9 non-HIV). Serum was collected in a tube without anticoagulant and underwent centrifugation at $4^{\circ} \mathrm{C}$ at setting $1500 \mathrm{~g}$ for $10 \mathrm{~min}$ utes. Briefly, serum was diluted with PBS and successively centrifuged at $2000 \mathrm{~g}$ for 10 minutes and $10,000 \mathrm{~g}$ for 30 minutes. The supernatant was then ultracentrifuged at 100,000 $g$ for 70 minutes using an SW-28 rotor (Beckman-Coulter) to pellet the sEV. Pellets were washed with PBS, centrifuged again at 100,000 $g$ for 70 minutes, and either resuspended in PBS for nanoparticle tracking analysis, immunoblotting, nanoparticle tracking analysis, and electron microscopy or resuspended in TRIzol reagent (Thermo Fisher Scientific) for RNA isolation and subsequent miRNA profiling. For the sEV isolation of mouse serum, blood obtained by cardiac puncture sat for 30 minutes at room temperature to allow coagulation prior to centrifuging twice at $500 \mathrm{~g}$ for 5 minutes and $2000 \mathrm{~g}$ for 10 minutes to remove cells and cellular debris. Supernatant was mixed 1:4 with double-filtered PBS and centrifuged at 10,000 g for 30 minutes to remove large vesicles. Supernatants containing serum sEVs were mixed in pools, each consisting of 10,000 g derived supernatants from 2 females and 1 male of the same genotype, having the same age distribution in all control and aDicer-KO samples. These pools were ultracentrifuged at 100,000g for 70 minutes using an SW-28 rotor. The pellets containing the sEVs were washed with double-filtered PBS and centrifuged again at $100,000 \mathrm{~g}$ for 70 minutes. After removing all supernatant, pellets were resuspended in TRIzol and proceeded to RNA isolation, miRNA retrotranscription, and PCR assay as described below. 
Nanoparticle tracking analysis. Vesicle concentration and size distribution was determined by the dynamic light scattering technology using a Nanosight LM10 (Malvern Panalytical) at the Nanosight Nanoparticle Sizing \& Quantification Facility at Massachusetts General Hospital (Charlestown, Massachusetts, USA). The script was programmed to take 5 videos for 30 seconds each for each sample. Samples were diluted 1:50 prior to running Nanosight system. For the calculation of the $\mathrm{sEV}$ concentration, this dilution and the volume of original serum where they were isolated from were taken into account.

Immunoblotting. Serum sEV and human 293 T cells (Takara catalog 632180) were resuspended in RIPA lysis buffer (MilliporeSigma) containing $0.1 \%$ SDS and protease and phosphatase inhibitors (Biotool) and incubated on ice for 30 minutes prior to centrifugation at 12,000 $\mathrm{g}$ for 10 minutes. Supernatants were used for Western blotting in SDS-PAGE electrophoresis. Protein concentration was determined by a BCA kit (Thermo Fisher Scientific). The following antibodies were used: TSG101 (sc-7964, Santa Cruz Biotechnology), CD63 (ab68418, Abcam), and calnexin (ab22595, Abcam).

Oil red $O$ staining. Human adipocytes were grown, differentiated, and transfected against Ltbp2, Nebl, Wisp2, and the triple combination as described above. Cells were washed with PBS and fixed with 4\% paraformaldehyde for 5 minutes. Cells were washed with PBS again and incubated for 1 hour with oil red $\mathrm{O}$ (ORO) working solution. To make ORO working solution, ORO stock solution ( $0.5 \%$ ORO in isopropanol) was mixed 3:2 with water and filtered. After 1-hour incubation, cells were washed with water, and representative photographs were taken immediately. Quantification of ORO signal was performed by ImageJ (NIH). Briefly, red/green/blue channels were separated in 3 pictures. Signal from red-containing pictures was quantified by selecting a $0-50$ threshold, and the percentage of positive area was later calculated.

Electron microscopy. CD63 immunogold staining of sEV preparations was performed at Electron Microscopy Facility at Harvard Medical School. sEVs were isolated by ultracentrifugation as described above and adsorbed to a hydrophilic carbon coated grid. After blocking with BSA, grids were incubated with antibody against the sEV marker CD63 (BD Pharmingen 556019) and later an IgG secondary antibody (Abcam ab6709). The antibody complex was detected using Protein A-gold $(10 \mathrm{~nm})$. The grids were examined in a JEOL 1200EX transmission electron microscope, and images were recorded with an AMT 2k charge-coupled device camera.

$R N A$ isolation and miRNA profiling. Upon addition of chloroform, samples were centrifuged at $12,000 \mathrm{~g}$ for 15 minutes. The upper phase was collected and mixed with isopropanol, ammonium acetate $(250 \mathrm{mM})$, and RNA-grade glycogen $\left(1 \mu \mathrm{g} / \mathrm{mL}\right.$, Thermo Fisher Scientific) and incubated overnight at $-20^{\circ} \mathrm{C}$. Samples were then centrifuged at $12,000 \mathrm{~g}$ for 30 minutes, washed twice with $75 \%$ ethanol, and resuspended in nuclease-free water. The RNA concentration was assessed by NanoDrop, and equal amounts of RNA for each sample were used for miRNA profile analysis. This was accomplished using a quantitative real-time PCRbased kit (RA660A-1, System Biosciences) following manufacturer's instructions. For single detection of miRNAs, RNA was retrotranscribed using miRCURY LNA Starter kit (QIAGEN) and assessed by PCR using highly specific miRCURY LNA primers (QIAGEN).

RNA extraction from human adipocytes was performed as described for sEV RNA isolation. Reverse transcription was done using High Capacity Reverse transcription kit (Applied Biosystems, Thermo Fisher Scientific) following manufacturer's instructions.

RNA-Seq. The total-RNA samples from human adipocytes were quantified using an Agilent 4200 TapeStation instrument, with a corresponding Agilent TapeStation RNA assay. The resulting RNA integrity number scores and concentrations were taken into account for qualifying samples to proceed.

The samples were normalized to $200 \mathrm{ng}$ of input in $50 \mu \mathrm{L}(4 \mathrm{ng} / \mu \mathrm{L})$, and the mRNA was captured using oligo-dT beads as part of the KAPA mRNA HyperPrep workflow. cDNA synthesis, adapter ligation, and amplification were conducted subsequently as part of the same workflow. Following amplification, residual primers were eluted away using KAPA Pure Beads in a $0.63 \times$ SPRI-based cleanup.

The resulting purified libraries were run on an Agilent 4200 TapeStation instrument, with a corresponding Agilent High Sensitivity D1000 ScreenTape assay to visualize the libraries and check that the size and concentrations of the libraries matched the expected product. qPCR with the KAPA Library Quantification kit, which uses primers complementary to the sequencing flow-cell oligos, was run to confirm the functional concentration. Molarity values obtained from this assay were used to normalize all samples in equimolar ratio for 1 final pool. The pool was denatured and loaded onto an Illumina NextSeq 500 instrument, with a high-output 75-cycle kit to obtain single-end 75 bp reads. The pool was loaded at $1.6 \mathrm{pM}$, with $5 \%$ PhiX spiked in as a sequencing control. The base call files were demultiplexed through the Harvard Biopolymers Facility Genomics Core's pipeline, and the resulting FASTQ files were 
used in subsequent bioinformatic analysis. RNA-Seq data were deposited in the National Center for Biotechnology Information's Gene Expression Omnibus database under accession number GSE183822.

Statistics. Normality of distribution was determined using the Shapiro-Wilk test. Data are presented as mean \pm SEM or median and IQR, depending on the normality of the distribution. Categorical variables are reported as proportions. Primary demographic comparisons were made between all 3 groups, non-HIV, HIV nonlipodystrophic, and HIV lipodystrophic, by overall comparison among the group by the appropriate test: 1-way ANOVA or Kruskal-Wallis test. Univariate regression was performed using Spearman's correlation coefficient.

For miRNA profiling analysis, the data from 2 participants ( $1 \mathrm{HIV} /$ lipo and another HIV/nonlipo) were removed due to high degree of hemolysis that significantly affected the miRNA profile results (Supplemental Table 1). As an initial step, although 1113 different miRNAs were measured, only those with $\mathrm{Ct}$ more than 35 in at least 5 samples per group (997 miRNAs) were considered expressed and therefore kept for further analysis. Expression data for miRNA expression were normalized to the mean Ct of all miRNAs for each sample. All samples displayed a similar Ct distribution. miRNA nomenclature was based on miRBase database version 22 (https://www.mirbase.org). To discover differential miRNA expression among groups, we used Limma, an $\mathrm{R}$ package for linear modeling that powers differential expression analyses (54). The same statistical method was used for analysis of RNA-Seq data. For RNA-Seq, genes meeting criteria for an absolute fold change 1.5 and $P<0.05$ were initially considered significant (Supplemental Tables 2-7). For multiple comparisons related to miRNA and RNA-Seq, an FDR was then applied as indicated in each experiment. Statistical significance was defined as FDR $<0.05$ for sEV miRNA profiling. Data for Ltbp2 expression in HIV nonlipodystrophic and lipodystrophic abdominal subcutaneous and dorsocervical adipose tissues were obtained from data set repository GSE28073 (20). For gene expression analysis, statistical significance was performed by 1-way ANOVA test followed by Dunnett's test comparing each group with the control group, controlling for multiple comparisons. Gene target prediction of miRNAs was performed using TargetScan and Diana-Tools software $(55,56)$, selecting a score more than 85 . For hierarchical cluster analysis, moderated $F$ test was used to detect genes that were differentially expressed between any 2 groups (namely control, miR-20a-3p, anti-miR-186, and anti-miR-324-5p). A total of 1031 genes were selected that had FDR $<0.05$ in the $F$ tests. Hierarchical cluster analysis was conducted based on the Euclidean distance of these selected genes. We defined 8 clusters according to the hierarchical tree. All statistical analyses were performed using SAS JMP (version 15), SPSS (version 20, IBM), and R.

Study approval. All animal studies were conducted in compliance with the regulations and ethics guidelines of the NIH and were approved by the IACUC of the Joslin Diabetes Center. The human study was approved by the Mass General Brigham Institutional Review Board. All individuals provided written informed consent prior to inclusion in the study.

\section{Author contributions}

SS designed the study, recruited patients, analyzed data, and drafted and critically reviewed the manuscript. SS was assigned as primary co-first author due to patient recruitment and conceptualization of the project. RGM designed the study, analyzed data, and drafted and critically reviewed the manuscript. MT analyzed data and critically reviewed the manuscript. KVF recruited patients and critically reviewed the manuscript. ARC analyzed data and critically reviewed the manuscript. CRK analyzed data and critically reviewed the manuscript. SKG designed the study, analyzed data, and drafted and critically reviewed the manuscript.

\section{Acknowledgments}

The investigators would like to thank the nursing staff on the MGH Translational and Clinical Research Center for their dedicated patient care and the volunteers who participated in this study. We thank Hui Pan and Jonathan Dreyfuss from Joslin Bioinformatics and Biostatistics Core for their help with data analysis. We thank Ashley M. Ciulla and the Biopolymers Facility Genomics Core at Harvard Medical School for their expertise and instrument availability that supported this work. We thank Tova Meshulam from BNORC for providing expertise regarding human adipocyte culture and differentiation.

This independent research was supported by the Gilead Sciences Research Scholars Program in HIV. Funding was also provided by NIH K23 HL136262 to SS; Deutsche Forschungsgemeinschaft Fellowship (DFG GA2426/1-1) to RGM; NIH R01 DK082659 to CRK; NIH UL1 TR001102 to the Harvard 
Catalyst/Harvard Clinical and Translational Science Center from the National Center for Research Resources and National Center for Advancing Translational Sciences; NIH P30 DK040561, Nutrition and Obesity Research Center at Harvard; and NIH P30 DK046200, BNORC. Funding sources had no role in the design of the study, data analysis, or writing of the manuscript.

Address correspondence to: Steven K. Grinspoon, Metabolism Unit, Massachusetts General Hospital, 55 Fruit Street, 5LON207, Boston, Massachusetts 02114, USA. Phone: 617.724.9109, Email: sgrinspoon@ mgh.harvard.edu.

1. Hussain I, Garg A. Lipodystrophy syndromes. Endocrinol Metab Clin North Am. 2016;45(4):783-797.

2. Palella FJ Jr., et al. Anatomic fat depots and coronary plaque among human immunodeficiency virus-infected and uninfected men in the multicenter AIDS cohort study. Open Forum Infect Dis. 2016;3(2):ofw098.

3. Srinivasa S, et al. Relationship of visceral and subcutaneous adipose depots to markers of arterial injury and inflammation among individuals with HIV. AIDS. 2019;33(2):229-236.

4. Stanley TL, et al. Effect of tesamorelin on visceral fat and liver fat in HIV-infected patients with abdominal fat accumulation: a randomized clinical trial. JAMA. 2014;312(4):380-389.

5. Agarwal N, et al. HIV-1 Vpr induces adipose dysfunction in vivo through reciprocal effects on PPAR/GR co-regulation. Sci Transl Med. 2013;5(213):213ra164.

6. Torriani M, et al. Dysfunctional subcutaneous fat with reduced dicer and brown adipose tissue gene expression in HIV-infected patients. J Clin Endocrinol Metab. 2016;101(3):1225-1234.

7. Balasubramaniam M, et al. Are microRNAs important players in HIV-1 infection? An update. Viruses. 2018;10(3):E110.

8. Mori MA, et al. Altered miRNA processing disrupts brown/white adipocyte determination and associates with lipodystrophy. J Clin Invest. 2014;124(8):3339-3351.

9. Reis FC, et al. Fat-specific Dicer deficiency accelerates aging and mitigates several effects of dietary restriction in mice. Aging (Albany NY). 2016;8(6):1201-1222.

10. Joy T, et al. Relation of body composition to body mass index in HIV-infected patients with metabolic abnormalities. $J$ Acquir Immune Defic Syndr. 2008;47(2):174-184.

11. Bogorodskaya M, et al. Measures of adipose tissue redistribution and atherosclerotic coronary plaque in HIV. Obesity (Silver Spring). 2020;28(4):749-755

12. Bourgi K, et al. Inflammation and metabolic complications in HIV. Curr HIV/AIDS Rep. 2018;15(5):371-381.

13. Thomou T, et al. Adipose-derived circulating miRNAs regulate gene expression in other tissues. Nature. 2017;542(7642):450-455.

14. Mori MA, et al. Extracellular miRNAs: from biomarkers to mediators of physiology and disease. Cell Metab. 2019;30(4):656-673

15. Thery C, et al. Isolation and characterization of exosomes from cell culture supernatants and biological fluids. Curr Protoc Cell Biol. 2006; Chapter 3:Unit 3.22.

16. GeneCards. LTBP2 Gene (Protein Coding). Latent Transforming Growth Factor Beta Binding Protein 2. https://www.genecards. org/cgi-bin/carddisp.pl?gene=LTBP2. Accessed August 20, 2021.

17. Grunberg JR, et al. Overexpressing the novel autocrine/endocrine adipokine WISP2 induces hyperplasia of the heart, white and brown adipose tissues and prevents insulin resistance. Sci Rep. 2017;7:43515.

18. Hammarstedt A, et al. WISP2 regulates preadipocyte commitment and PPAR $\gamma$ activation by BMP4. Proc Natl Acad Sci U S A. 2013;110(7):2563-2568.

19. Gustafson B, et al. Restricted adipogenesis in hypertrophic obesity: the role of WISP2, WNT, and BMP4. Diabetes. 2013;62(9):2997-3004.

20. Sevastianova K, et al. Comparison of dorsocervical with abdominal subcutaneous adipose tissue in patients with and without antiretroviral therapy-associated lipodystrophy. Diabetes. 2011;60(7):1894-1900.

21. Shackleton S, et al. LMNA, encoding lamin A/C, is mutated in partial lipodystrophy. Nat Genet. 2000;24(2):153-156.

22. Caux F, et al. A new clinical condition linked to a novel mutation in lamins A and $\mathrm{C}$ with generalized lipoatrophy, insulin-resistant diabetes, disseminated leukomelanodermic papules, liver steatosis, and cardiomyopathy. J Clin Endocrinol Metab. 2003;88(3):1006-1013.

23. Bidault G, et al. LMNA-linked lipodystrophies: from altered fat distribution to cellular alterations. Biochem Soc Trans. 2011;39(6):1752-1757.

24. Casey Klockow L, et al. The HIV-1 protein Vpr targets the endoribonuclease Dicer for proteasomal degradation to boost macrophage infection. Virology. 2013;444(1-2):191-202.

25. Bennasser Y, Jeang KT. HIV-1 Tat interaction with Dicer: requirement for RNA. Retrovirology. 2006;3:95.

26. Torriani M, et al. Deiodinase 2 expression is increased in dorsocervical fat of patients with HIV-associated lipohypertrophy syndrome. J Clin Endocrinol Metab. 2012;97(4):E602-E607.

27. Cui J, et al. MicroRNA-20a-3p regulates the host immune response to facilitate the mycobacterium tuberculosis infection by targeting IKK $\beta / \mathrm{NF}-\mathrm{kB}$ pathway. Int Immunopharmacol. 2021;91:107286.

28. Sun JL, et al. MicroRNA regulation in hypoxic environments: differential expression of microRNAs in the liver of largemouth bass (Micropterus salmoides). Fish Physiol Biochem. 2020;46(6):2227-2242.

29. Stepien EL, et al. Circulating ectosomes: determination of angiogenic microRNAs in type 2 diabetes. Theranostics. 2018;8(14):3874-3890

30. Bukhari MMM, et al. Role of MicroRNAs in establishing latency of human immunodeficiency virus. Crit Rev Eukaryot Gene Expr. 2020;30(4):337-348.

31. Wu G, et al. MiRNA-324-5p inhibits inflammatory response of diabetic vessels by targeting CPT1A. Eur Rev Med Pharmacol Sci. 
2020;24(24):12836-12843.

32. Gu J, et al. Downregulated miRNA-324-5p aggravates neuronal injury induced by oxygen-glucose deprivation via modulating RAN. Exp Ther Med. 2020;19(1):658-664.

33. Peng Y, et al. SUFU mediates EMT and Wnt/ $\beta$-catenin signaling pathway activation promoted by miRNA-324-5p in human gastric cancer. Cell Cycle. 2020;19(20):2720-2733.

34. Bresciani E, et al. miRNA-218 targets Lipin-1 and glucose transporter type 4 genes in 3T3-L1 cells treated with lopinavir/ritonavir. Front Pharmacol. 2019;10:461.

35. Squillace N, et al. Changes in subcutaneous adipose tissue microRNA expression in HIV-infected patients. J Antimicrob Chemother. 2014;69(11):3067-3075.

36. Valadi $\mathrm{H}$, et al. Exosome-mediated transfer of mRNAs and microRNAs is a novel mechanism of genetic exchange between cells. Nat Cell Biol. 2007;9(6):654-659.

37. Skog J, et al. Glioblastoma microvesicles transport RNA and proteins that promote tumour growth and provide diagnostic biomarkers. Nat Cell Biol. 2008;10(12):1470-1476.

38. Bastard JP, et al. Association between altered expression of adipogenic factor SREBP1 in lipoatrophic adipose tissue from HIV-1-infected patients and abnormal adipocyte differentiation and insulin resistance. Lancet. 2002;359(9311):1026-1031.

39. Luzi L, et al. Intramyocellular lipid accumulation and reduced whole body lipid oxidation in HIV lipodystrophy. Am J Physiol Endocrinol Metab. 2003;284(2):E274-E280.

40. Haugaard SB, et al. Tumor necrosis factor alpha is associated with insulin-mediated suppression of free fatty acids and net lipid oxidation in HIV-infected patients with lipodystrophy. Metabolism. 2006;55(2):175-182.

41. van der Valk M, et al. Lipodystrophy in HIV-1-positive patients is associated with insulin resistance in multiple metabolic pathways. AIDS. 2001;15(16):2093-2100

42. Patni N, et al. Juvenile-onset generalized lipodystrophy due to a novel heterozygous missense LMNA mutation affecting lamin C. Am J Med Genet A. 2017;173(9):2517-2521.

43. Speckman RA, et al. Mutational and haplotype analyses of families with familial partial lipodystrophy (Dunnigan variety) reveal recurrent missense mutations in the globular C-terminal domain of lamin A/C. Am J Hum Genet. 2000;66(4):1192-1198.

44. Genschel J, Schmidt HH. Mutations in the LMNA gene encoding lamin A/C. Hum Mutat. 2000;16(6):451-459.

45. Caron M, et al. Some HIV protease inhibitors alter lamin A/C maturation and stability, SREBP-1 nuclear localization and adipocyte differentiation. AIDS. 2003;17(17):2437-2444.

46. Kannisto K, et al. Expression of adipogenic transcription factors, peroxisome proliferator-activated receptor gamma co-activator 1, IL-6 and CD45 in subcutaneous adipose tissue in lipodystrophy associated with highly active antiretroviral therapy. AIDS. 2003;17(12):1753-1762.

47. Lihn AS, et al. Increased expression of TNF-alpha, IL-6, and IL-8 in HALS: implications for reduced adiponectin expression and plasma levels. Am J Physiol Endocrinol Metab. 2003;285(5):E1072-E1080.

48. Asensi V, et al. IL-1beta (+3954C/T) polymorphism could protect human immunodeficiency virus (HIV)-infected patients on highly active antiretroviral treatment (HAART) against lipodystrophic syndrome. Genet Med. 2008;10(3):215-223.

49. Lee TS, Chau LY. Heme oxygenase-1 mediates the anti-inflammatory effect of interleukin-10 in mice. Nat Med. 2002;8(3):240-246.

50. Piantadosi CA, et al. Heme oxygenase-1 couples activation of mitochondrial biogenesis to anti-inflammatory cytokine expression. J Biol Chem. 2011;286(18):16374-16385.

51. Gurung C, et al. Dicer represses the interferon response and the double-stranded RNA-activated protein kinase pathway in mouse embryonic stem cells. J Biol Chem. 2021;296:100264.

52. Mori MA, et al. Role of microRNA processing in adipose tissue in stress defense and longevity. Cell Metab. 2012;16(3):336-347.

53. Singh AM, et al. Human beige adipocytes for drug discovery and cell therapy in metabolic diseases. Nat Commun. 2020;11(1):2758.

54. Ritchie ME, et al. Empirical array quality weights in the analysis of microarray data. BMC Bioinformatics. $2006 ; 7: 261$.

55. Agarwal V, et al. Predicting effective microRNA target sites in mammalian mRNAs. Elife. 2015;4.

56. Paraskevopoulou MD, et al. DIANA-microT web server v5.0: service integration into miRNA functional analysis workflows. Nucleic Acids Res. 2013;41(web server issue):W169-W173 